ÉTAT DES LIEUX

DE LA CONSOMMATION

DES BEnZODIAZÉPINES

En FRAnCE

JAMUIER 2012
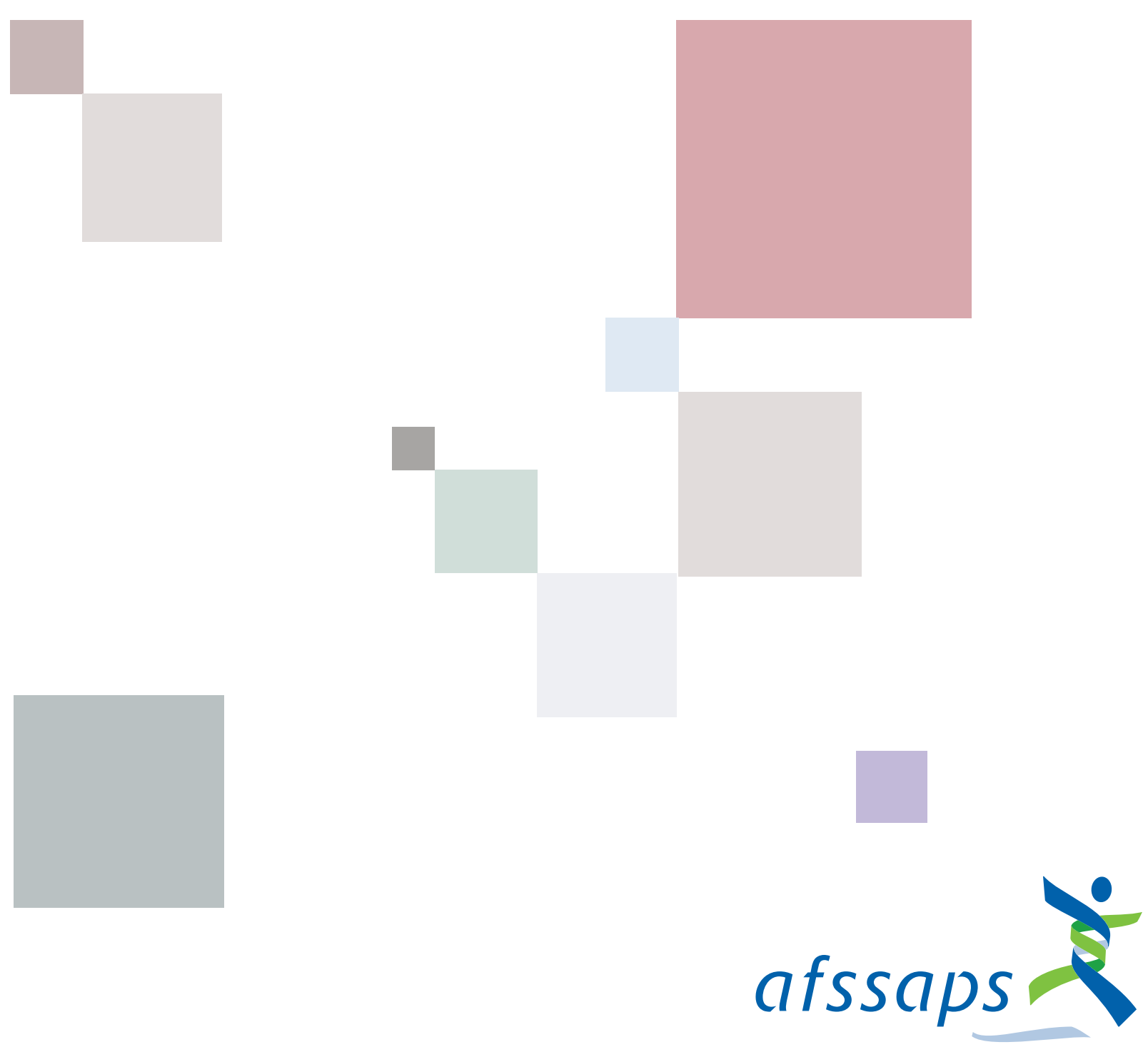


\section{Quelques chiffres}

$\rightarrow 22$ benzodiazépines ou apparentées sont commercialisées en France en 20II;

$\rightarrow 134$ millions de boîtes vendues en 2010 dont $50,2 \%$ d'anxiolytiques et 37,6 \% d'hypnotiques;

$\rightarrow 183$ millions d'euros de chiffres d'affaires en 2010 , soit $0,7 \%$ du montant total des ventes de médicaments en France;

$\rightarrow$ En 2010, $20 \%$ de la population française a consommé au moins une fois une benzodiazépine ou apparentée;

$\rightarrow 60 \%$ des consommateurs de benzodiazépines ou apparentées sont des femmes;

Le temps de traitement médian est de 7 mois pour une benzodiazépine anxiolytique et hypnotique ou apparentée;

$\rightarrow$ Environ la moitié des sujets traités par une benzodiazépine anxiolytique et hypnotique le sont plus de 2 ans (avec ou sans interruption de traitement);

Environ la moitié des patients sous benzodiazépine hypnotique (ou apparentée) ou anxiolytique ne bénéficie que d'une seule déliurance/prescription.

\section{La rédaction de ce rapport a été coordonnée par Philippe Cavalié et nathalie Richard}

\section{Ont participé à la rédaction:}

- Tiphaine Canarelli

- Cédric Collin

- Marie-Anne Courné

- Bernard Delorme

- Catherine Messina-Courlot

- Christel Saussier

nous remercions Bernard Bégaud, Maryse Lapeyre-Mestre, Claude Magnin et Pierre Polomeni pour leur relecture attentive de ce rapport. 


\section{Résumé}

Les benzodiazépines sont des molécules qui agissent sur le système nerveux central et qui possèdent toutes des propriétés anxiolytiques, hypnotiques, myorelaxantes et anticonvulsivantes mais à des niveaux différents en fonction de leur structure chimique. En France, 22 benzodiazépines et apparentées sont actuellement commercialisées. Elles sont ainsi indiquées et prescrites, selon leurs propriétés pharmacologiques, dans le traitement symptomatique des manifestations anxieuses, dans les manifestations du sevrage alcoolique, dans les troubles sévères du sommeil, dans le traitement des épilepsies généralisées ou partielles et le traitement des contractures musculaires douloureuses.

Depuis les années 1990, de nombreux travaux ont souligné le niveau élevé de la consommation de médicaments psychotropes des français, en particulier des anxiolytiques et des hypnotiques, principalement représentés par les benzodiazépines. En 2009, selon certaines données européennes, la France était le deuxième pays européen consommateur d'anxiolytiques (après le Portugal) et d'hypnotiques laprès la Suède). Par ailleurs, un certain nombre d'effets négatifs et de risques sont associés à la consommation de benzodiazépines

Ce rapport vise à étudier la consommation actuelle des benzodiazépines en France et son évolution depuis 10 ans au travers des données de ventes déclarées à l'Afssaps, de données de remboursement de l'assurance maladie, d'études réalisées sur des populations particulières et de données provenant des systèmes de vigilance de l'Afssaps. II présente également les actions réalisées par les autorités sanitaires ainsi qu'un plan d'action proposant des mesures pour limiter la consommation des benzodiazépines et favoriser leur bon usage.

Chaque année, un français sur cinq (20 \%) consomme au moins une benzodiazépine ou une molécule apparentée. Ainsi, plus de 25 millions de sujets ont été exposés à une benzodiazépine entre mi-2006 et mi-20II.

Depuis 2002, la consommation des benzodiazépines anxiolytiques a diminué avec un nombre de consommateurs constant, suggérant une diminution de la consommation individuelle. On observe cependant une tendance à la hausse depuis 2009. La consommation des benzodiazépines hypnotiques et apparentées et le nombre de consommateurs sont restés stables. La consommation des benzodiazépines hypnotiques diminue au profit d'une augmentation régulière de celle des substances apparentées aux benzodiazépines (zolpidem et zopiclone).

Les benzodiazépines sont prescrites essentiellement par les médecins généralistes.

Les sujets consommant des benzodiazépines sont majoritairement des femmes (près de $60 \%$ ). La consommation des anxiolytiques et des hypnotiques augmente avec l'âge. Les femmes les plus jeunes consomment principalement des benzodiazépines anxiolytiques et le tétrazépam, les plus âgées les anxiolytiques et les hypnotiques. Les hommes les plus jeunes consomment préférentiellement le tétrazépam, les plus âgés les hypnotiques et les anxiolytiques. II n'existe pas de profil différent de consommation entre benzodiazépines anxiolytiques et hypnotiques dont le temps de traitement médian est de 7 mois. Au cours du suivi de cinq ans de patients traités, $52 \%$ des sujets traités sont exposés plus de 2 ans à une benzodiazépine anxiolytique et hypnotique, $46 \%$ pour le clonazépam et $30 \%$ pour le tétrazépam. Dans 21,I \% des cas, le traitement par benzodiazépine est associé à un traitement antidépresseur et dans 4,9\% des cas, à un traitement antipsychotique. De plus, $21 \%$ des patients ont eu au moins une fois un traitement par plusieurs benzodiazépines en même temps. 
Le traitement par les benzodiazépines est indispensable pour de nombreux patients mais présente aussi des risques. Les benzodiazépines peuvent en particulier entraîner des troubles de la mémoire et du comportement, une altération de l'état de conscience et des fonctions psychomotrices. Ces effets sont accrus chez le sujet âgé. De plus, plusieurs études suggèrent un lien possible entre benzodiazépines, démences dont la maladie d'Alzheimer en particulier les travaux d'une équipe de recherche dont les résultats seront prochainement publiés. Par rapport à cette problématique, dix études internationales ont déjà été publiées mais les résultats ne sont pas homogènes. Cinq études concluent à un lien, une conclut à un effet protecteur des benzodiazépines sur l'apparition d'une démence et quatre ne retrouvent pas de lien ou ne permettent pas de la mettre en évidence pour des raisons statistiques

L'usage de benzodiazépines expose également à un risque de dépendance psychique et physique qui s’accompagne d'un syndrome de seurage à l'arrêt du traitement.

À côté de ces effets indésirables, le réseau d'addictovigilance a mis en évidence une utilisation problématique des benzodiazépines avec un usage abusif ou détourné par les toxicomanes et un risque d'usage criminel à des fins de soumission chimique.

Enfin, il a été largement démontré que la consommation de benzodiazépines altérait les capacités à conduire un véhicule. Une étude pharmaco-épidémiologique, soutenue par l'Afssaps, a confirmé qu'une part non négligeable des accidents de la route pouvait leur être attribuée.

L'importance de la consommation de benzodiazépines en France et les risques qui leur sont liés ont conduit les autorités sanitaires à mettre en place des mesures visant à favoriser leur bon usage. Ces mesures sont axées sur l'amélioration de l'information des professionnels de santé et des patients, l'encadrement de la prescription et de la déliurance, la prévention du risque d'abus et d'usage détourné et la surveillance par les réseaux de vigilance sanitaire.

Le degré d'exposition élevé et persistant de la population française aux benzodiazépines et certaines études suggérant un possible lien entre leur consommation prolongée et la survenue de démences dont la maladie d'Alzheimer doit inciter non seulement à développer la surveillance et la recherche dans ce domaine mais aussi à modifier l'usage extensif fait de ces molécules en France, au demeurant nécessaires à la santé de très nombreux patients.

De nouvelles actions doivent ainsi être envisagées et renforcées, fondées sur les données de pharmacovigilance, d'addictovigilance et de pharmaco-épidémiologie. 


\section{Sommaire}

I. Les benzodiazépines en 20II 7

2. Panorama européen 9

3. Données de consommation nationale 12

3.I. La consommation de benzodiazépines en France 12

3.I.I. La situation en $2010 \quad 12$

3.1.2. Les génériques $\quad 14$

3.1.3. L'évolution 2000-2010 14

3.2. Population exposée à une benzodiazépine ou apparentée à partir des données de l'Assurance maladie $\quad 18$

4. Données d'exposition 20

4.I. Caractéristiques démographiques des patients à partir des données de l'Assurance maladie $\quad 20$

4.2. Co-morbidités et traitements associés 21

4.3. Modalités de traitement par une benzodiazépine 22

5. Les risques liés à l'usage des benzodiazépines 25

5.I. Les risques 25

5.I.I. Les risques généraux 25

5.1.2. Les associations avec d'autres médicaments ou d'autres substances 26

5.I.3. Les risques liés à une utilisation dans des situations particulières 26

5.I.4. L'usage problématique 29

6. Les actions des autorités sanitaires 30

6.I. Les actions réalisées 30

6.I.I. Amélioration de l'information des professionnels de santé et des patients $\quad 30$

6.I.2. Encadrement de la prescription et de la déliurance 30

6.l.3. Prévention du risque de soumission chimique et d'abus 31

6.I.4. Surveillance sanitaire 31

6.2. Les pistes d'amélioration 31

7. Références bibliographiques 33

8. Annexes 36 


\section{Données de contexte}

Les médicaments psychotropes recouvrent un ensemble de molécules: les neuroleptiques, les antidépresseurs, les anxiolytiques et les hypnotiques; ces deux dernières classes étant principalement représentées par les benzodiazépines.

Commercialisées depuis les années 60 , les benzodiazépines possèdent des propriétés anxiolytiques, sédatives, myorelaxantes mais aussi anticonvulsivantes. Elles présentent une structure chimique commune formée d'un cycle diazépine fusionné avec un cycle benzène. Des radicaux varient d'une molécule à l'autre ce qui leur confère leur activité spécifique et des paramètres pharmacocinétiques différents (délai d'action, durée d'action, temps d'élimination...). Elles agissent sur le système nerveux central par l'intermédiaire des récepteurs CABA.

Deux molécules hypnotiques sont également rapprochées de cette classe : le zolpidem et la zopiclone. Bien qu'elles n'aient pas la même structure chimique, elles possèdent un mécanisme d'action et des effets proches des benzodiazépines. Elles sont donc dites « apparentées aux benzodiazépines ».

Ainsi, les 22 benzodiazépines et apparentées actuellement commercialisées en France sont indiquées, selon leur propriété principale, dans le traitement de l'anxiété, des troubles sévères du sommeil, de l'épilepsie ou des contractures musculaires douloureuses.

De nombreux travaux et études portant sur les médicaments psychotropes ont souligné, depuis les années 1990, le niveau élevé de leur consommation en France, en particulier pour les molécules anxiolytiques et hypnotiques principalement représentées par les benzodiazépines [I-4]. En 2000, le chiffre d'affaires des vingt-deux benzodiazépines s'élevait à 260 millions d'euros et représentait 1,5\% du montant des ventes. En 2010, ce chiffre d'affaires était de 183 millions d'euros, soit 0,7\% du montant total des ventes réalisées en France. Cependant, entre 2000 et 2010 bien que la consommation des anxiolytiques ait diminué, la consommation des hypnotiques est restée stable et celles du clonazépam et du tétrazépam ont augmenté.

Selon le rapport de l'office parlementaire d'évaluation des politiques de santé publiê en 2006, 15 à $20 \%$ des français avaient un usage ponctuel des anxiolytiques et hypnotiques et $10 \%$ un usage régulier. La part de la population ayant pris un médicament psychotrope au cours des douze derniers mois (environ $25 \%$ ) serait deux fois plus élevée que la moyenne des pays européens limitrophes de la France [5].

Compte tenu de l'importance de la consommation des benzodiazépines en France, de leur profil de sécurité d'emploi et de certains usages problématiques, les benzodiazépines font, depuis de nombreuses années, l'objet d'une attention particulière par les autorités sanitaires et notamment par l'Afssaps. De nombreuses mesures ont ainsi été déjà prises pour améliorer le bon usage, maîtriser la consommation et éviter l'usage détourné de ces molécules.

Le degré d'exposition élevé et persistant de la population française à ces molécules, la publication récente de plusieurs études et rapports suggérant un lien possible entre ces molécules et la survenue de démences invitent l'Afssaps à une nouvelle mobilisation pour préparer un plan renforcé d'actions ayant pour objectif d'optimiser l'utilisation de ces molécules très utiles à de nombreux patients.

Ce rapport en est un élément visant à étudier, de façon synthétique, l'évolution de la consommation en France au cours de ces dernières années. 


\section{Les benzodiazépines en 20|I}

Vingt-deux benzodiazépines - ou apparentées - sont actuellement commercialisées en France.

Benzodiazépines et apparentées actuellement commercialisés en France.

\begin{tabular}{|c|c|c|c|c|}
\hline Substance active & $\begin{array}{l}\text { nom des spécialités } \\
\text { commercialisées }\end{array}$ & Classe & $\begin{array}{l}\text { Demi-vie } \\
\text { (h) }\end{array}$ & $\begin{array}{l}\text { Conditionnements } \\
\text { des formes orales }\end{array}$ \\
\hline \multicolumn{5}{|l|}{ Anxiolytiques } \\
\hline Alprazolam & Xanax et génériques & Anxiolytique & $10-20$ & 30 comprimés \\
\hline Bromazépam & Lexomil et génériques & Anxiolytique & 20 & 30 comprimés \\
\hline Clobazam* & Urbanyl & Anxiolytique & 20 & $\begin{array}{l}30 \text { gélules ( } 5 \text { mg) } \\
30 \text { comprimés (I0 et } 20 \text { mg) }\end{array}$ \\
\hline $\begin{array}{l}\text { Clorazépate } \\
\text { potassique }\end{array}$ & Tranxene & Anxiolytique & $30-150$ & $\begin{array}{l}30 \text { gélules ( } 5 \text { et } 10 \text { mg) } \\
28 \text { gélules ( } 20 \text { mg) }\end{array}$ \\
\hline Clotiazépam & Veratran & Anxiolytique & 4 & 30 comprimés \\
\hline Diazépam & Valium & Anxiolytique & $32-47$ & $\begin{array}{l}30 \text { comprimés ( } 10 \mathrm{mg} \text { ) } \\
40 \text { comprimés ( } 2 \text { et } 5 \mathrm{mg}) \\
\text { flacon } 20 \mathrm{ml} \text { solution buvable }\end{array}$ \\
\hline Ethyl loflazépate & Victan & Anxiolytique & 77 & 30 comprimés \\
\hline Lorazépam & Temesta et génériques & Anxiolytique & $10-20$ & 30 comprimés \\
\hline nordazépam & nordaz & Anxiolytique & $30-150$ & 30 comprimés \\
\hline Oxazépam & Seresta et génériques & Anxiolytique & 8 & $\begin{array}{l}30 \text { comprimés (I0mg) } \\
20 \text { comprimés (50mg) }\end{array}$ \\
\hline Prazépam & Lysanxia et génériques & Anxiolytique & $30-150$ & $\begin{array}{l}40 \text { comprimés (10 mg) } \\
20 \text { comprimés ( } 40 \text { mg) } \\
\text { flacon } 20 \mathrm{ml} \text { solution buvable }\end{array}$ \\
\hline \multicolumn{5}{|l|}{ Hypnotiques } \\
\hline Estazolam & nuctalon & Hypnotique & 17 & 20 comprimés \\
\hline Flunitrazépam & Rohypnol, narcozep & Hypnotique & $16-35$ & 7 comprimés \\
\hline Loprazolam & Havlane & Hypnotique & 8 & 20 comprimés \\
\hline Lormétazépam & noctamide & Hypnotique & 10 & 14 comprimés \\
\hline midazolam & $\begin{array}{l}\text { Hypnovel et génériques } \\
\text { Versed }\end{array}$ & Hypnotique & $1,5-2,5$ & $\begin{array}{l}\text { formes injectables } \\
\text { uniquement }\end{array}$ \\
\hline nitrazépam & Mogadon & Hypnotique & $16-48$ & 20 comprimés \\
\hline Témazépam & normison & Hypnotique & $5-8$ & $\begin{array}{l}14 \text { comprimés }(10 \text { mg) } \\
7 \text { comprimés }(20 \text { mg) }\end{array}$ \\
\hline \multicolumn{5}{|c|}{ Apparentēs aux benzodiazépines } \\
\hline Zolpidem & Stilnox et génériques & Hypnotique & $0,7-3,5$ & 7 et 14 comprimés \\
\hline Zopiclone & Imovane et génériques & Hypnotique & 5 & 5 et 14 comprimés \\
\hline \multicolumn{5}{|l|}{ myorelaxant } \\
\hline Tétrazépam & Myolastan et génériques & Myorelaxant & $18-26$ & 20 comprimés \\
\hline \multicolumn{5}{|l|}{ Anticonvulsivant } \\
\hline Clonazépam & Rivotril & Antiépileptique & $20-60$ & $\begin{array}{l}28 \text { comprimés } \\
\text { flacon } 20 \mathrm{ml} \text { solution buvable }\end{array}$ \\
\hline
\end{tabular}

Demi-vie courte < $10 \mathrm{~h}$ : exemple: midazolam, témézépam, clotiazépam.

Demi-vie intermédiaire 10-24 h: exemple: alprazolam, bromazépam, clobazam, estazolam,

lorazépam, nitrazépam, tétrazépam.

Demi-vie longue > $24 \mathrm{~h}$ : exemple : clonazépam, diazépam, éthyl loflazépate.

* Le Clobazam dispose également pour deux de ses dosages (I0 et 20 mg) d'indications dans le traitement de l'épilepsie généralisée ou partielle. L'Oms l'a cependant classé parmi les anxiolytiques. Dans ce rapport il a été traité exclusivement comme anxiolytique. 
Même si elles partagent la même activité pharmacodynamique, elles ne possèdent pas toutes les mêmes indications et elles relèvent donc de plusieurs classes pharmaco-thérapeutiques(II):

- le tétrazépam est un myorelaxant d'action centrale (m03B) : il est indiqué pour le traitement des contractures musculaires douloureuses en rhumatologie;

- le clonazépam est classé parmi les antiépileptiques (n03) et ses indications sont limitées au traitement des épilepsies généralisées ou partielles;

- onze benzodiazépines sont classées parmi les anxiolytiques (n05B). Elles possèdent toutes les deux indications suivantes:

- traitement symptomatique des manifestations anxieuses sévères et/ou invalidantes,

- prévention et traitement du delirium tremens et des autres manifestations du sevrage alcoolique;

- les neuf dernières sont utilisées comme hypnotiques et sédatifs (n05C). La plupart de ces benzodiazépines ont des indications limitées aux troubles sévères du sommeil dans les cas d'insomnie occasionnelle ou d'insomnie transitoire. Quelques-unes possèdent cependant des indications relatives à la sédation rapide ou à l'anesthésie.

(I) La classification utilisée est la classification ATC (anatomique, thérapeutique, chimique), établie par l'Organisation Mondiale de la Santé afin de favoriser des études internationales sur l'utilisation des médicaments. Les médicaments sont classés selon l'organe sur lequel ils agissent et/ou leurs caractéristiques thérapeutiques et chimiques. La classification se décline en cinq niveaux: hiveau I groupe « anatomique », niveau 2 groupe « thérapeutique », niveau 3: sous-groupe « thérapeutique/pharmacologique », niveau 4 : sous-groupe « chimique/thérapeutique/pharmacologique », hiveau 5 : sous-groupe « substance chimique » 


\section{Panorama européen}

Quelle que soit la classe thérapeutique étudiée, peu de données internationales sont aisément accessibles et lorsqu'elles sont disponibles, ces données peuvent différer d'une source à l'autre. Les sources de données de consommation européenne sont hétérogènes. II peut s’agir des données de vente, des données statistiques provenant des organismes payeurs (systèmes nationaux d'assurance maladie) ou bien de données de déliurance par les pharmacies. Les données de remboursement peuvent conduire à une sous-estimation de la consommation réelle. L'amplitude des erreurs liées à l'utilisation de sources de données différentes est difficile à estimer. Les résultats sont donc à interpréter avec précaution. Deux pays voisins de la France sont absents des données présentées : la Belgique et l'Italie. La consommation des médicaments en Belgique est estimée à partir des données de remboursements; or, les anxiolytiques et les hypnotiques ne sont pas remboursables dans ce pays. En Italie, les données ne sont pas publiées.

Par ailleurs, les données disponibles concernent l'ensemble des hypnotiques et des anxiolytiques, qu'ils s'agissent des benzodiazépines et des autres classes pharmacologiques (sédatifs pour les hypnotiques par exemple).

Les données de consommation identifiées ont été rapportées au nombre de doses définies journalières (DDJ) pour 1000 habitants par jour, soit le nombre de patients pour 1000 habitants et par jour.[6] Établie sous l'égide de l'OmS, la Dose Définie Journalière constitue une posologie de référence qui ne reflète pas nécessairement la posologie recommandée par l'Amm ni la posologie effective : elle constitue un étalon de mesure. L'usage des DDJ élimine ainsi les difficultés de mesure liées à l'hétérogénéité des tailles de conditionnement et de dosage des médicaments commercialisés. Pour tenir compte des différences de population d'un pays à l'autre, le nombre de DDJ est divisé par le nombre total d'habitants. Par convention, les résultats sont présentés pour mille habitants et par jour (DDJ/I 000H/J). Cet indicateur rend donc possible les comparaisons de consommations et permet de calculer, le cas échéant, une consommation moyenne internationale. Son interprétation doit cependant être prudente et il convient en particulier de ne pas établir de corrélation entre l'évolution de la consommation et celle du nombre de patients exposés. Ainsi, la baisse du nombre de DDJ peut correspondre à une moindre consommation individuelle sans que le nombre total de consommateurs ait pour autant diminué.

Parmi les pays étudiés, la France est le deuxième pays consommateur d'anxiolytiques, soit environ 50 DDJ (après le Portugal, 80 DDJ) et d'hypnotiques, soit 35 DDJ (après la Suède, 52 DDJ) en 2009 (voir figures I et 2).

La consommation d'anxiolytiques est stable en France (benzodiazépines, carbamates et autres types de molécules), en Suède et en Allemagne alors qu'elle augmente au Portugal et en Espagne et qu'elle diminue dans des proportions variables au Danemark, aux Pays-Bas et au Royaume-Uni (figure 3) [6].

La consommation d'hypnotiques reste relativement stable chez nos voisins à l'exception des Pays-Bas et du Danemark (figure 4) [6].

La baisse observée aux Pays-Bas, tant pour les anxiolytiques que pour les hypnotiques, appelle un commentaire particulier. En effet, un remboursement conditionnel lié aux pathologies traitées a été mis en place en 2009. Ainsi, $70 \%$ des prescriptions d'hypnotiques et d'anxiolytiques antérieures à la réforme ne sont plus prises en charge comme l'illustrent les figures I et 2, mais le niveau de consommation n'a pas diminué dans les proportions attendues, selon le Stichting Farmaceutische Kengetallen (Fondation néerlandaise pour les statistiques pharmaceutiques). 
Figure 1: Miveaux de consommation des anxiolytiques (nO5B) dans certains pays européens, en DDJ/I 000 hab/J en 2009

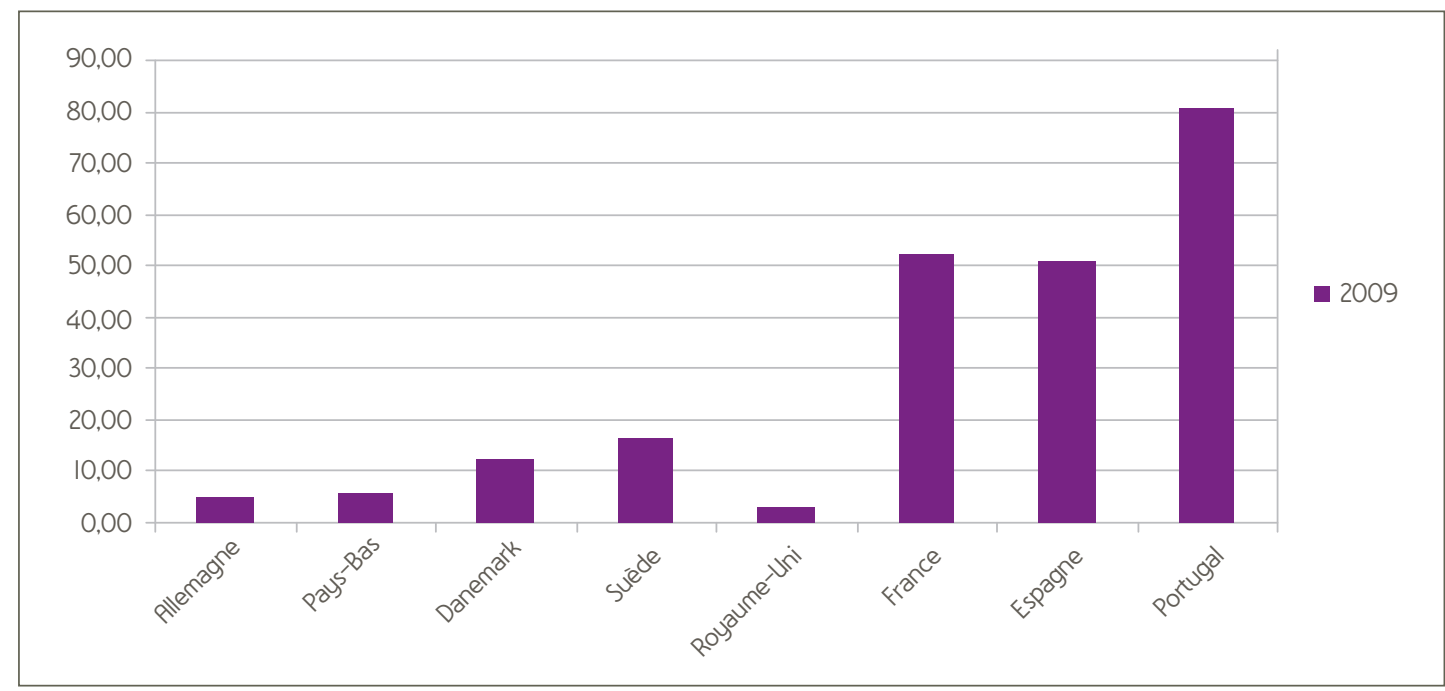

Figure 2: Miveaux de consommation des hypnotiques (n05C) dans certains pays européens, en DDJ/I 000 hab/J en 2009

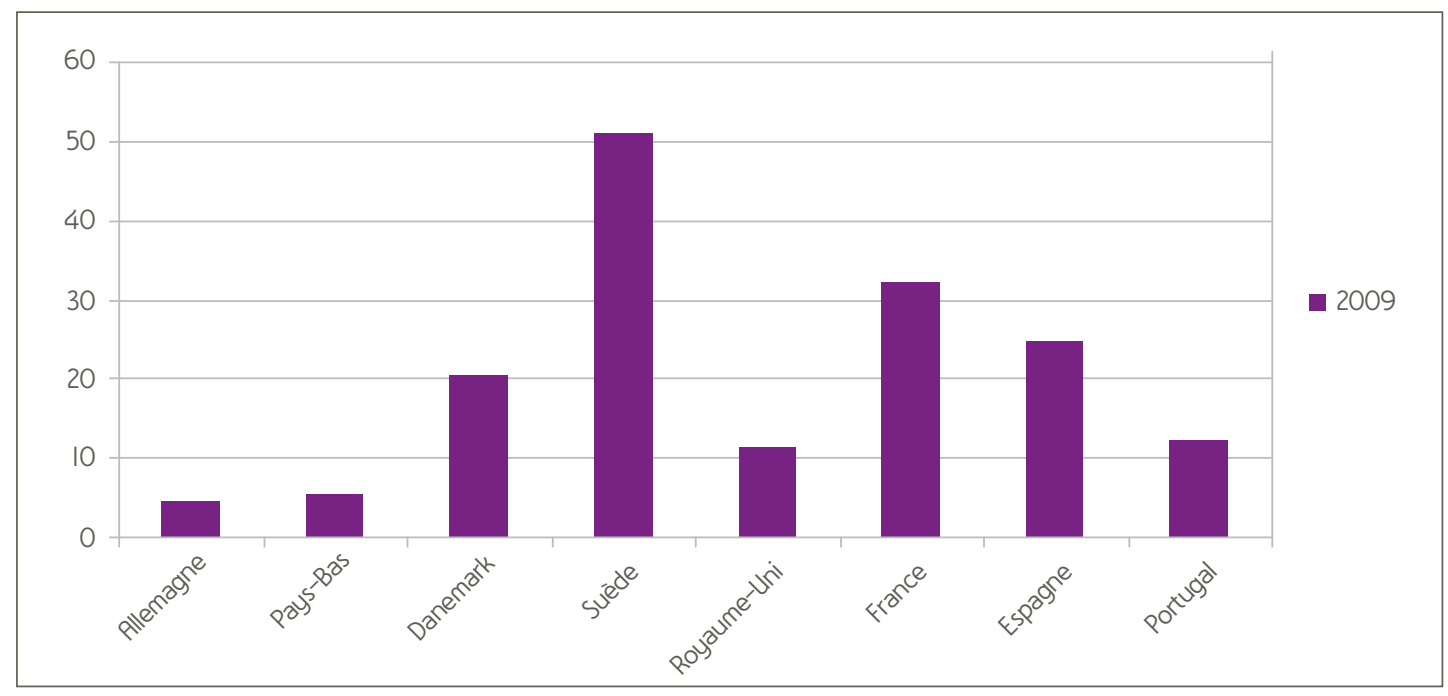


Figure 3: Evvolution des niveaux de consommation des anxiolytiques (n05B) dans certains pays européens, en DDJ/I 000 hab./J, 2005-2009

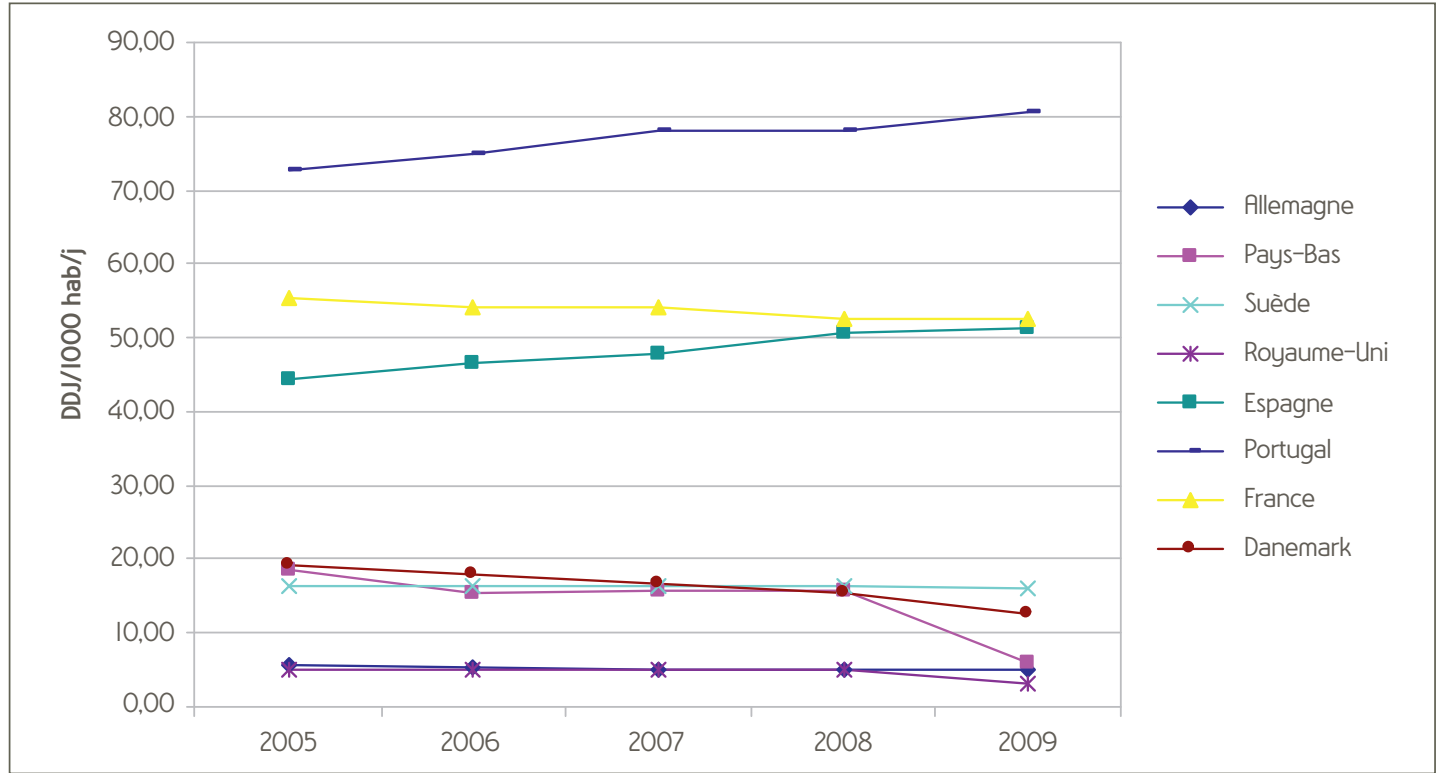

Figure 4: Évolution des niveaux de consommation des hypnotiques (n05C) dans certains pays européens, en DDJ/I 000 hab./J, 2005-2009

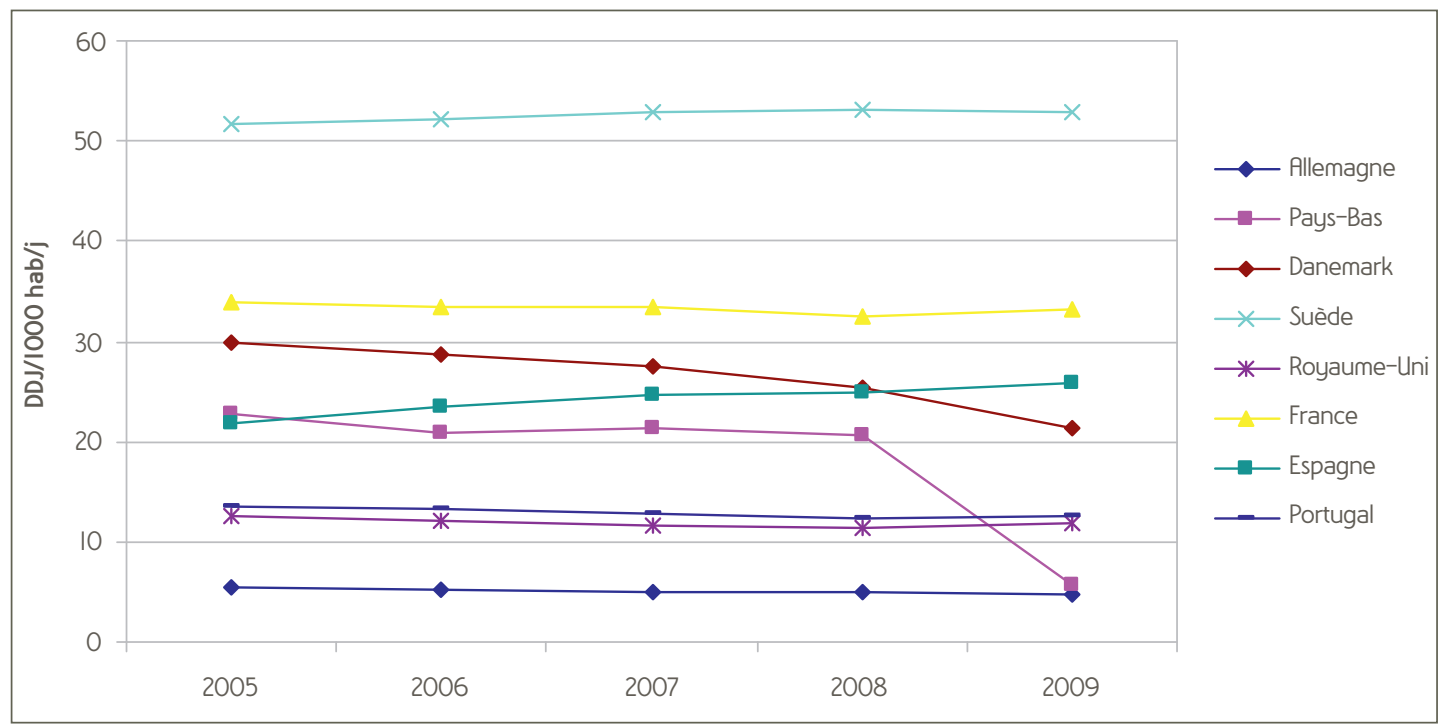




\section{Données de consommation nationale}

\section{En 2010:}

- 64,9 millions de boîtes d'anxiolytiques dérivés de la benzodiazépine ont été vendues en ville. Ces molécules représentaient, en $2010,83,3 \%$ de la consommation totale d'anxiolytiques exprimée en nombre de DDJ;

- 48,2 millions de boîtes d'hypnotiques dérivés ou apparentés aux benzodiazépines ont été vendues en ville. Ces neuf molécules représentaient en $201076,3 \%$ de la consommation totale d'hypnotiques et de sédatifs exprimée en nombre de DDJ;

- 9,7 millions de boîtes de tétrazépam ont été vendues en ville. Le tétrazépam représentait 57,9 \% de la consommation totale, exprimée en nombre de DDJ, des myorelaxants d'action centrale;

- 5,9 millions de boîtes de clonazépam ont été vendues en ville. Le clonazépam représentait I2,5 \% de la consommation totale d'antiépileptiques, exprimée en nombre de doses définies journalières.

\section{I. La consommation de benzodiazépines en France}

\section{I.I. La situation en 2010}

La consommation des vingt-deux benzodiazépines actuellement commercialisées en France se répartit inégalement au sein des quatre classes pharmaco-thérapeutiques où elles sont présentes. Les aspects méthodologiques détaillant les sources de données sont présentés en annexe I. Les anxiolytiques représentaient en 2010 plus de la moitié des boîtes de benzodiazépines vendues, suivis par les hypnotiques $(37,6 \%)$.

Les parts respectives des myorelaxants et des antiépileptiques demeurent relativement faibles mais il faut relever, dans l'un et l'autre cas:

- qu’une seule benzodiazépine est disponible dans chacune de ces deux classes;

- leur usage augmente;

- ces deux molécules, en raison, de leurs indications, ne sont pas toujours perçues comme des benzodiazépines (que les patients associent souvent au traitement de l'anxiété et des troubles du sommeil).

Figure 5: Répartition des indications de benzodiazépines en 2010

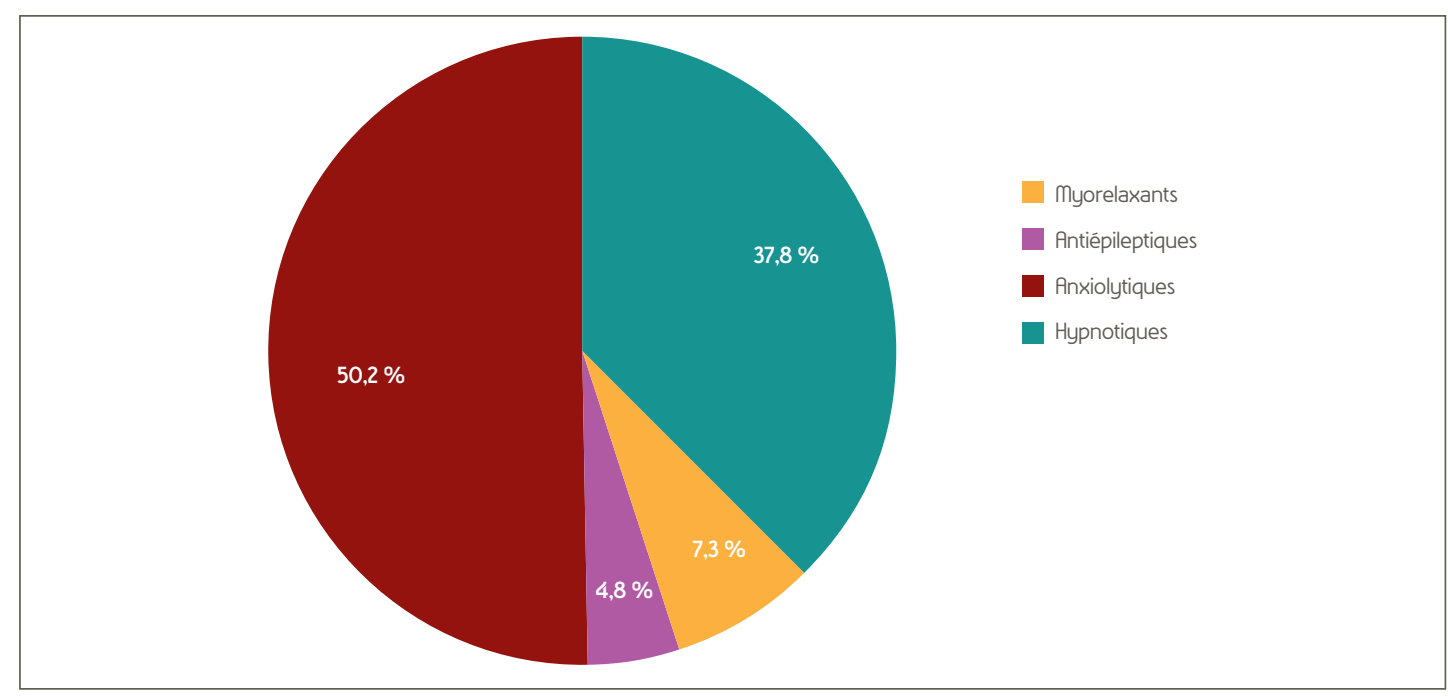


Au total, ces vingt-deux benzodiazépines représentaient I34 millions de boîtes, soit 3,8 \% de la consommation totale de médicaments en 20I0. Ce pourcentage est élevé car il faut tenir compte du fait qu'il s'agit:

- de médicaments soumis à une prescription médicale obligatoire;

- de médicaments qui ne sont pas destinés à des traitements chroniques, mais au contraire de médicaments dont la durée de traitement est, pour la plupart d'entre eux, étroitement limitée: 12 semaines pour les anxiolytiques et 4 semaines pour les hypnotiques.

Leur chiffre d'affaires global s'élevait en 2010 à 183 millions d'euros (en prix fabricant hors taxes), soit $0,7 \%$ des ventes totales de médicaments en France en 20I0. L'écart entre la part en valeur et la part en quantités s'explique par le fait que les benzodiazépines sont des médicaments peu onéreux, pour la plupart génériqués. Les prix publics TTC de ces médicaments varient entre I, $43 €$ et 5,35€, avec une majorité de prix compris entre 2 et 3 euros. II s'agit donc de médicaments dont le prix est nettement inférieur à celui d'autres spécialités remboursables, toutes classes confondues. Tous ces médicaments sont pris en charge par les régimes d'assurance maladie au taux de $65 \%$, à l'exception du tétrazépam déremboursé depuis le ler décembre 20ll.

Les benzodiazépines sont majoritairement utilisées en ville : sur les 134 millions de boîtes consommées en 20I0, I28 ont été vendues en officines et 6 ont été utilisées par les établissements hospitaliers.

Tableau I: Benzodiazépines et apparentées commercialisées

\begin{tabular}{|c|c|c|c|c|}
\hline Substance active & Classe & $\begin{array}{c}\text { nombre de boîtes } \\
\text { vendues } 2010\end{array}$ & $\begin{array}{l}\text { nombre d'unité } \\
\text { « standard » } 2010\end{array}$ & $\begin{array}{l}\text { Chiffre d'affaires } \\
\text { France } 2010\end{array}$ \\
\hline Zolpidem & Hypnotique & 25 & 362 & 29 \\
\hline Alprazolam & Anxiolytique & 19 & 567 & 17 \\
\hline Zopiclone & Hypnotique & 17 & 248 & 22 \\
\hline Bromazépam & Anxiolytique & 13 & 408 & 19 \\
\hline Lorazépam & Anxiolytique & 10 & 309 & 10 \\
\hline Tétrazépam & myorelaxant & 10 & 196 & 23 \\
\hline Oxazépam & Anxiolytique & 9 & 235 & 14 \\
\hline Clonazépam & Antiépileptique & 6 & 146 & 7 \\
\hline Prazépam & Anxiolytique & 6 & 213 & 10 \\
\hline Lormétazépam & Hypnotique & 5 & 67 & 6 \\
\hline Clorazépate potassique & Anxiolytique & 4 & III & 10 \\
\hline Diazépam & Anxiolytique & 3 & 83 & 3 \\
\hline Clobazam & Anxiolytique & 2 & 61 & 4 \\
\hline Loprazolam & Hypnotique & 1 & 29 & 3 \\
\hline Midazolam & Hypnotique & 1 & 7 & 2 \\
\hline Flunitrazépam & Hypnotique & 0,6 & 4 & 0,3 \\
\hline Clotiazépam & Anxiolytique & 0,6 & 17 & 0,7 \\
\hline nordazépam & Anxiolytique & 0,6 & 17 & 1 \\
\hline Éthyl loflazépate & Anxiolytique & 0,5 & 14 & 1 \\
\hline nitrazépam & Hypnotique & 0,4 & 8 & 0,3 \\
\hline Témazépam & Hypnotique & 0,4 & 3 & 0,4 \\
\hline Estazolam & Hypnotique & 0,3 & 6 & 0,4 \\
\hline Total & & 134 & $3 \| 3$ & 183 \\
\hline
\end{tabular}


Si l'on tient compte des différences dans les tailles de conditionnement en convertissant le nombre de

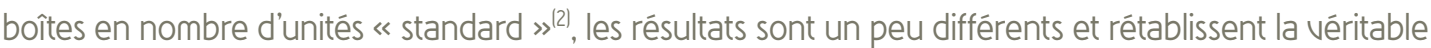
hiérarchie: ce sont les anxiolytiques qui sont les plus fréquemment utilisés.

\section{I.2. Les génériques}

Commercialisées depuis des dizaines d'années (les premières benzodiazépines ont été autorisées à la fin des années 60), la plupart des vingt-deux benzodiazépines mises sur le marché en France disposent de génériques. Dans la majorité des cas, la part de marché désormais détenue par leurs génériques est importante:

- en 20I0, les génériques représentaient $93 \%$ de la consommation exprimée en nombre de DDJ de tétrazépam; en 2000, $28 \%$;

- le clonazépam n’a pas de générique autorisé à ce jour en France;

- parmi les anxiolytiques dérivés de la benzodiazépine, les génériques représentaient 40,9\% de la consommation exprimée en nombre de DDJ en 20I0, contre 5,2 \% en 2000;

- parmi les benzodiazépines hypnotiques et sédatives, les génériques représentaient 6I,I \% en 2010 et $4,3 \%$ en 2000 .

\subsubsection{L'évolution 2000-2010}

Le niveau de consommation des benzodiazépines en 2010 ne peut être correctement analysé et interprété qu'à la lumière des résultats des années précédentes. En effet, des actions ont été menées et des mesures adoptées pour favoriser, d'une part, le bon usage de ces molécules et pour limiter, d'autre part, leur usage abusif et détourné: ont-elles eu un impact sur le niveau de consommation de ces médicaments?

Figure 6: Consommation totale de benzodiazépines et apparentées de 2000 à 2010 (DDJ/l 000 hab/j)

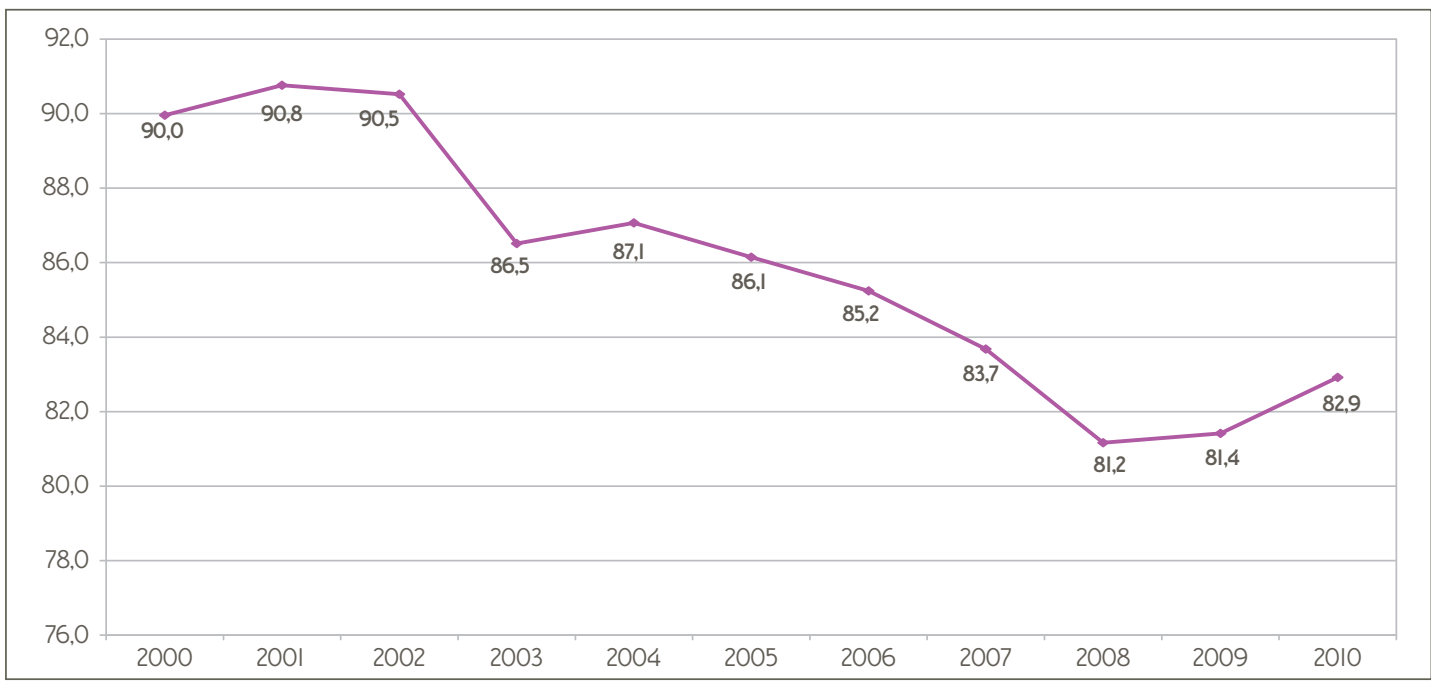

La consommation a globalement diminué, comme le montre la figure 6 , dont le détail est présenté dans le tableau de l'annexe II.

(2) Qui correspond à la plus petite dose commune d'un produit : gélule, ampoule, sachet, etc. 
Cette évolution, qui peut être jugée satisfaisante, mérite cependant d'être nuancée pour deux raisons

- une tendance à la reprise se manifeste, surtout perceptible en 20I0,

- la diminution des consommations ne concerne pas l'ensemble des molécules et des classes, ainsi les consommations de tétrazepam et de clonazépam augmentent.

\section{- Le tétrazépam}

Figure 7: Consommation de tétrazépam de 2000 à 2010 (DDJ/l 000 hab/j)

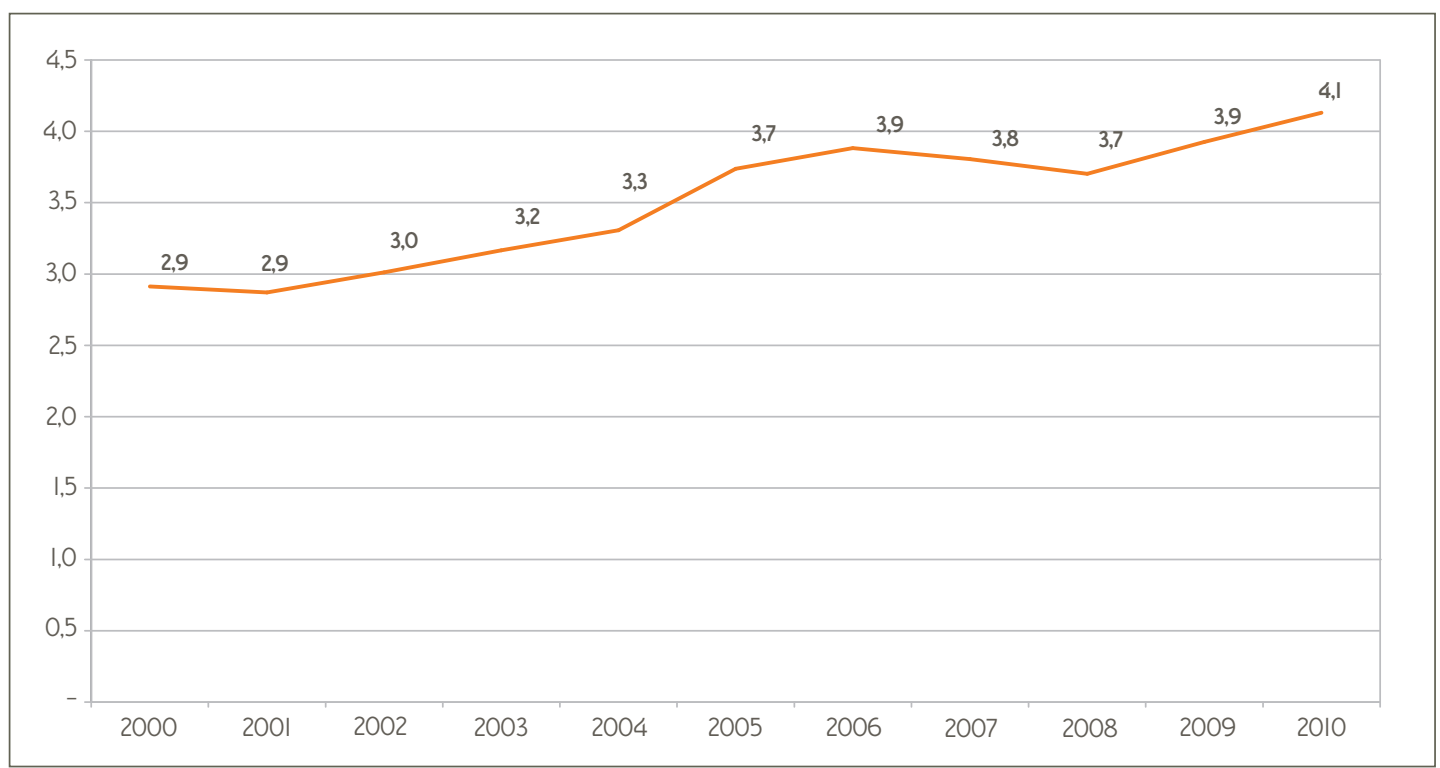

Prescrit comme myorelaxant d'action centrale, le tétrazépam est principalement utilisé en médecine ambulatoire: la consommation hospitalière de ce médicament ne représentait que $2,5 \%$ en 2010 . Au cours de ces dix dernières années, l'usage de cette molécule a progressé au rythme de $+3,6 \%$ en moyenne annuelle (sur la base de la consommation exprimée en nombre de DDJ pour 1000 habitants et par jour). Après la phase de stabilisation observée entre 2005 et 2008, la consommation a de nouveau augmenté. La baisse de son taux de remboursement (15\% en auril 2010 et déremboursé en décembre 20II) ne semble avoir exercé aucun impact notable sur le niveau de ses ventes. Le tétrazépam représentait $57,9 \%$ de la consommation totale, exprimée en nombre de DDJ, des myorelaxants d'action centrale 


\section{- Le clonazépam}

Figure 8: Consommation de clonazépam de 2000 à 2010 (DDJ/I 000 hab/j)

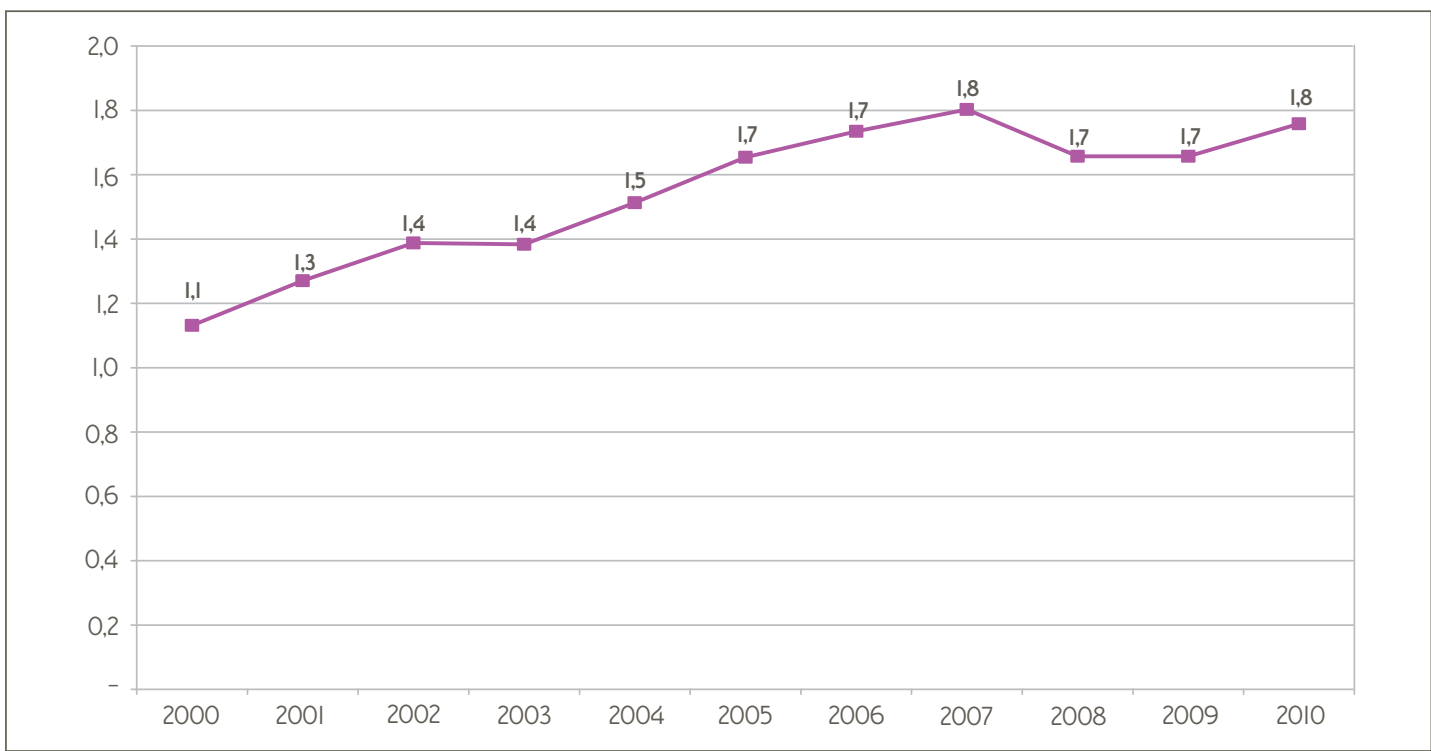

La consommation de clonazépam a également augmenté et sa courbe d'évolution - quoique légèrement plus accentuée -présente un profil assez proche de celle du tétrazépam, avec une période de stabilisation suivie par une tendance à la reprise. En moyenne annuelle, la consommation a augmenté de 4,5\%. L'usage hospitalier est un peu plus important que celui du tétrazépam mais ne représentait que 7,8 \% de la consommation en 20I0. Le clonazépam représentait 12,5\% de la consommation totale d'antiépileptiques, exprimée en nombre de doses définies journalières.

\section{- Les benzodiazépines prescrites comme anxiolytiques}

Figure 9: Consommation de benzodiazépines anxiolytiques de 2000 à 2010 (DDJ/I 000 hab/j)

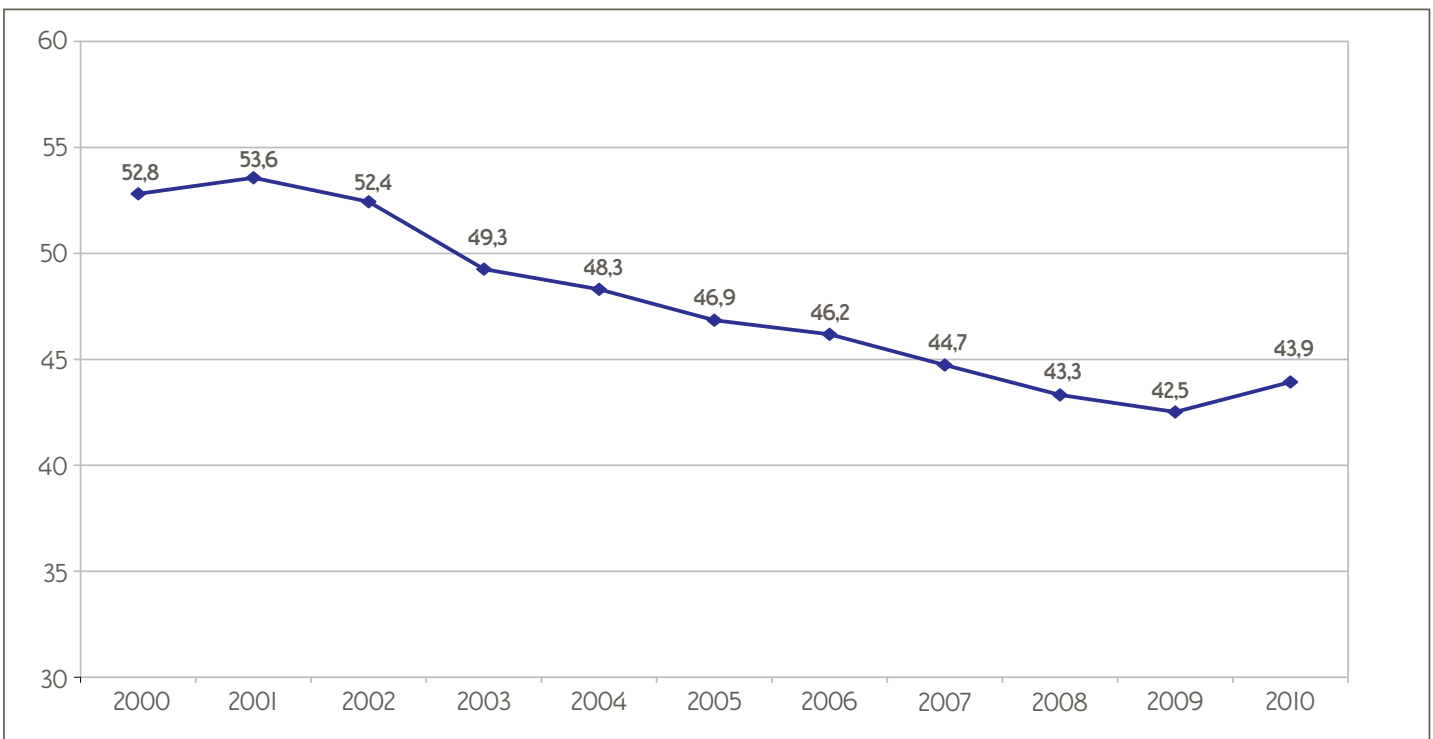


La consommation des onze benzodiazépines utilisées comme anxiolytiques a diminué au cours de ces dix dernières années: en moyenne -I, 8 \% par an. Amorcé en 2002, ce mouvement de baisse s'est néanmoins interrompu en 2010 où l'on observe une reprise de la consommation. Ce retournement de tendance - qui deura bien sûr être confirmé par les résultats de 20ll - ne peut pas être imputé à un report des prescriptions d'une autre classe d'anxiolytiques (carbamates par exemple) vers les benzodiazépines. En effet, la consommation d'anxiolytiques - toutes familles confondues - a augmenté en 2010. À cet égard, il est précisé que les benzodiazépines représentaient en 20I0, 83,3 \% de la consommation totale d'anxiolytiques exprimée en nombre de DDJ.

Si l'on ne considère que les seules molécules benzodiazépines, il apparaît que la consommation était fortement concentrée en 20I0. En effet, trois d'entre elles - le lorazépam, le bromazépam et l'alprazolam - représentaient $61 \%$ de la consommation de benzodiazépines anxiolytiques.

Par ailleurs,l'usage hospitalier demeure limité et représentait en 2010, 6,3\% de la consommation de benzodiazépines anxiolytiques.

\section{- Les benzodiazépines prescrites comme hypnotiques}

Figure 10: Consommation de benzodiazépines hypnotiques et apparentées de 2000 à 2010 (DDJ/I 000 hab/j)

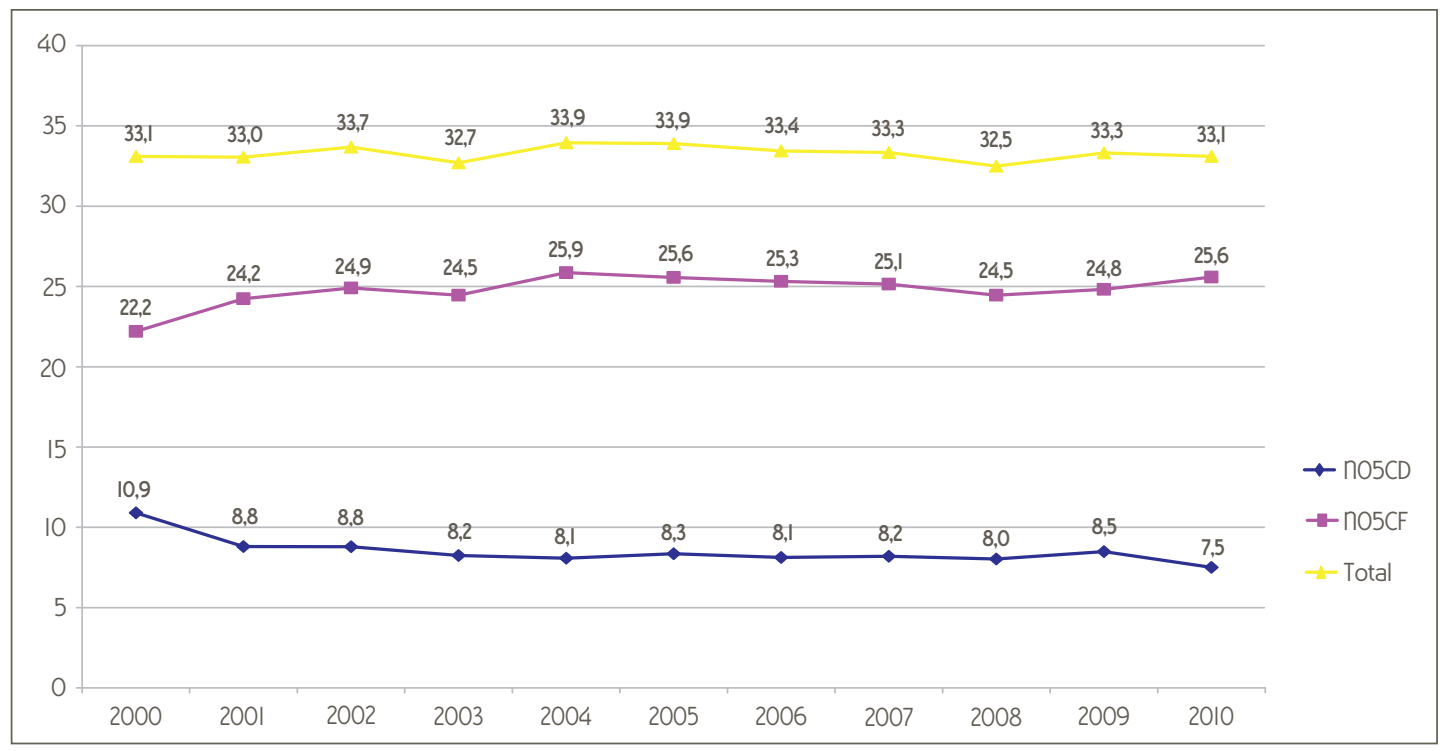

La diminution régulière de la consommation des dérivés de la benzodiazépine (n05CD) a eu pour contrepartie une augmentation de celle des substances apparentées aux benzodiazépines (n05CF), entraînant une stabilité globale de la classe considérée. Méanmoins, le niveau global de cette consommation peut apparaître très élevé puisque la prescription de ces médicaments est limitée à quatre semaines, alors que celle des anxiolytiques est limitée à douze semaines. On pourrait donc s'attendre a priori à ce que l'écart entre le niveau de consommation des anxiolytiques et celui des hypnotiques soit beaucoup plus important qu'il ne l'est en réalité. La part de la consommation hospitalière était, en 2010, de 6,9\%. Deux molécules, la zopiclone et le zolpidem, sont très largement utilisées et représentent plus de 77 \% de la consommation française. Le midazolam, qui n'est pas indiqué dans les troubles du sommeil mais utilisé comme anesthésique ou pour assurer une sédation rapide, représentait plus de 14 \% de la consommation des médicaments de ces deux classes en 2010 . 
Au total, ces neuf molécules représentaient en 2010 76,3\% de la consommation totale d'hypnotiques et de sédatifs exprimée en nombre de DDJ. L'évolution de la consommation des benzodiazépines et apparentées de 2000 à 2010 figure dans l'annexe II.

\subsection{Population exposée à une benzodiazépine ou apparentée à partir des données de l'Assurance maladie}

L'échantillon généraliste des bénéficiaires est un échantillon au I/97e des sujets affiliés au régime général de l'assurance maladie lainsi qu'au régime agricole et au régime des salariés indépendants depuis 20ll), soit environ un peu plus de 520000 bénéficiaires en 2010. L'ECB intègre ainsi les sujets consommateurs de soins (dont les médicaments) mais également non consommateurs.

On définit le taux de prévalence comme le nombre de cas total présent /d'une maladie, de l'exposition à un médicament...) à un instant « $t$ » et dans une population déterminée.

Le nombre de bénéficiaires affiliés au régime général ayant eu au moins un remboursement d'une benzodiazépine au cours de la période du ler juillet 2006 au 30 juin 20II est de 212548.

En 20I0, 105364 patients sélectionnés dans l'échantillon des bénéficiaires ont perçu au moins un remboursement de benzodiazépines ou apparentées quelle que soit l'indication; soit une estimation de 10,2 millions de sujets affiliés au régime général de l'assurance maladie, soit finalement environ 14 millions de français.

Le taux de prévalence annuelle d'exposition à au moins une benzodiazépine ou une apparentée en 2010 est estimé à 20,0 \% pour les sujets affiliés au régime général de l'assurance maladie. La prévalence annuelle a également été estimée en 2007,2008 et 2009. La figure II reprend l'évolution de la prévalence annuelle d'exposition à une benzodiazépine ou apparentée de 2007 à 2010 . Elle est stable au cours du temps quelle que soit l'indication de la benzodiazépine, soit II \% de la population affiliée au régime général pour une benzodiazépine anxiolytique, 6,5 \% pour une benzodiazépine ou apparenté hypnotique, I,6 \% pour le clonazépam, et plus de 6,5\% pour le tétrazépam dont la prévalence a augmenté de 6,4 \% entre 2007 et 2010

Figure II: Évolution du taux de prévalence annuelle d'exposition à une benzodiazépine ou apparentée de 2007 à 2010 pour les sujets affiliés au régime général de l'Assurance maladie

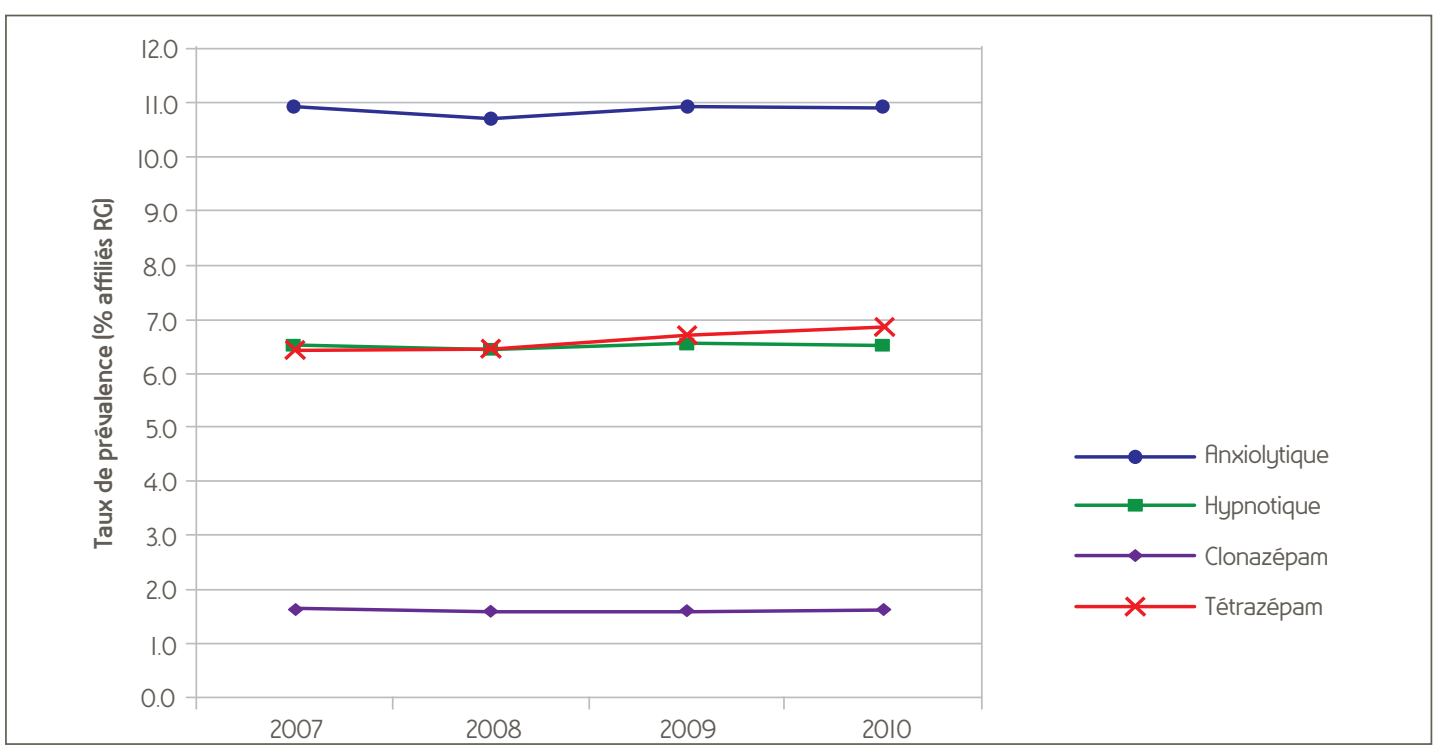


Les benzodiazépines et apparentées les plus consommées au cours de l'année 2009 et 2010 sont présentés dans la figure 12. Parmi les 10,2 millions de sujets exposés, 3,5 millions consomment du tétrazépam, 2 millions consomment du bromazépam et 2 millions du zolpidem. Ce sont les 3 molécules les plus consommées. De surcroît, le nombre de leurs bénéficiaires a légèrement augmenté entre 2009 et $20101+6,1 \%$, $+1,4 \%$ et $+4,3 \%$ respectivement).

Ensuite, les benzodiazépines et apparentées les plus consommées en 2010 sont l'alprazolam lenviron I,6 million de consommateurs) et la zopiclone (environ I,2 million de consommateurs). L'alprazolam présente l'augmentation la plus marquée du nombre de sujets consommant des benzodiazépines entre 2009 et $2010(+\mid 0,4 \%)$.

Figure 12: Mombre de patients prévalents affiliés au régime général de l'assurance maladie par benzodiazépines ou apparentées en 2009 et 2010

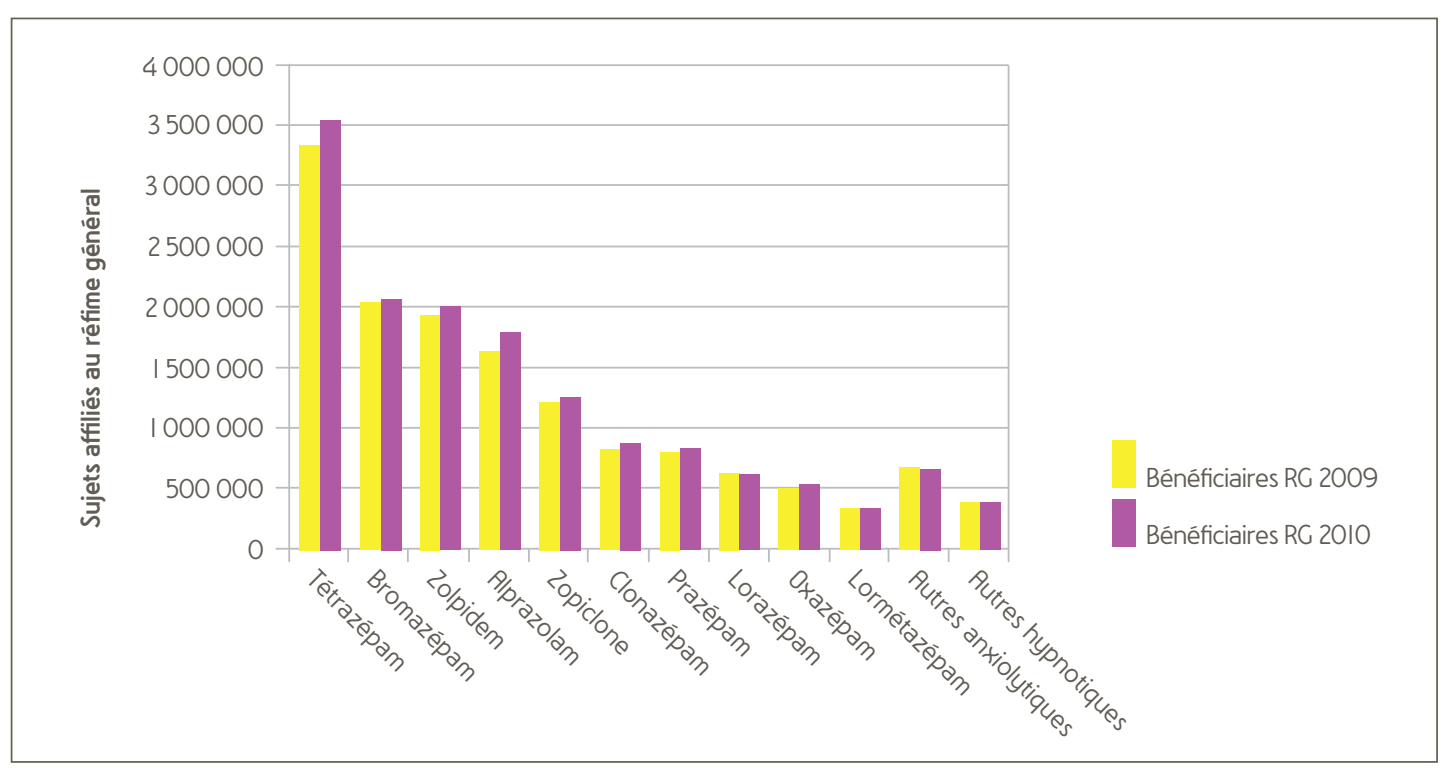

Le nombre de sujets bénéficiant d'au moins un remboursement de benzodiazépines ou apparentées est stable au cours des 4 dernières années, malgré une diminution de la consommation exprimée au travers des données de ventes. Ce résultat suggère donc une diminution de la consommation de benzodiazépines au niveau individuel (diminution de la posologie ou diminution du temps de traitement). 


\section{Données d'exposition}

L'analyse a été effectuée sur l'ensemble des bénéficiaires sélectionnés entre le ler juillet 2006 et le 30 juin 20II, soit une période de 5 ans.

\section{4.l. Caractéristiques démographiques des patients à partir des données de l'Assurance maladie}

Ce sont l'âge et le sexe, directement disponibles à partir de la date de naissance du bénéficiaire présent dans l'échantillon des bénéficiaires.

L'âge médian des patients bénéficiant d'un traitement par benzodiazépines, toutes classes confondues, est de 48 ans $^{(3)}$ : les sujets consommant des benzodiazépines anxiolytiques ont un âge médian de 50 ans et ceux consommant des benzodiazépines hypnotiques ont un âge médian de 55 ans. II est à noter que les consommateurs de tétrazépam sont beaucoup plus jeunes avec un âge médian de 42 ans (Annexe III, Table All. En effet, le tétrazépam, principalement indiqué dans les contractures musculaires, est souvent prescrit à des sujets sportifs d'âge moyen plus faible que celui de la population générale.

\section{$59,3 \%$ de ces patients sont des femmes.}

41,I \% des patients traités par benzodiazépines, le sont par une benzodiazépine anxiolytique, 36,2 \% par le tétrazépam, 18,3\% par une benzodiazépine hypnotique ou apparentées et 4,4\% par le clonazépam.

À noter que près de $3 \%$ des sujets qui prennent une benzodiazépine anxiolytique ont moins de 18 ans (seuls 0,9\% des moins de 18 ans bénéficient d'une benzodiazépine hypnotique ou apparentées et 2,I \% pour le tétrazépam et clonazépam).

L'âge médian des hommes qui consomment des benzodiazépines est de 47 ans ${ }^{(4)}$, celui des femmes est de 49 ans $^{(5)}$. Comme l'illustre la figure 13 , la prévalence de patients consommant des benzodiazépines anxiolytiques augmente fortement avec l'âge et majoritairement chez les femmes. Près d'une femme sur cinq consomme des benzodiazépines anxiolytiques lorsqu'elle est âgée de 30 à 40 ans et près d'une femme sur trois lorsqu'elle est âgée de 70 à 75 ans. Cette augmentation est plus modérée chez l'homme, avec près de $10 \%$ des hommes qui consomment une benzodiazépine anxiolytique lorsqu'ils sont âgés de 30 à 40 ans et moins de $15 \%$ lorsqu'ils ont entre 70 et 75 ans. La prévalence de sujets consommant des benzodiazépines hypnotiques et apparentées varie entre 5 et $10 \%$ selon le sexe, lorsque les sujets sont âgés entre 30 et 40 ans et augmente plus chez les femmes avec l'âge lenviron $18 \%$ de 70 à 75 ans, vs. II \% chez les hommes). La prévalence de patients traités par tétrazépam augmente jusqu'à 30-40 ans avant de se stabiliser et de diminuer à partir de 50-60 ans. La prévalence de patients traités par clonazépam augmente très légèrement avec l'âge.

Les femmes les plus jeunes consommant des benzodiazépines consomment principalement des anxiolytiques et le tétrazépam, les plus âgées des anxiolytiques et des hypnotiques. Les hommes consomment préférentiellement du tétrazépam, des anxiolytiques puis des hypnotiques lorsqu'ils sont jeunes, jusqu'à 30-40 ans, puis préférentiellement des hypnotiques et des anxiolytiques après 50 ans.

(3) Intervalle interquartile (IQR) : 25-75e percentile : 35-62 ans.

(4) IQR: $35-60$ ans

(5) IQR: 36-64 ans 
Figure 13: Taux de prévalence* de sujets traités par BZD, pour 100 habitants en France, en fonction de l'âge, du sexe et de l'indication du traitement

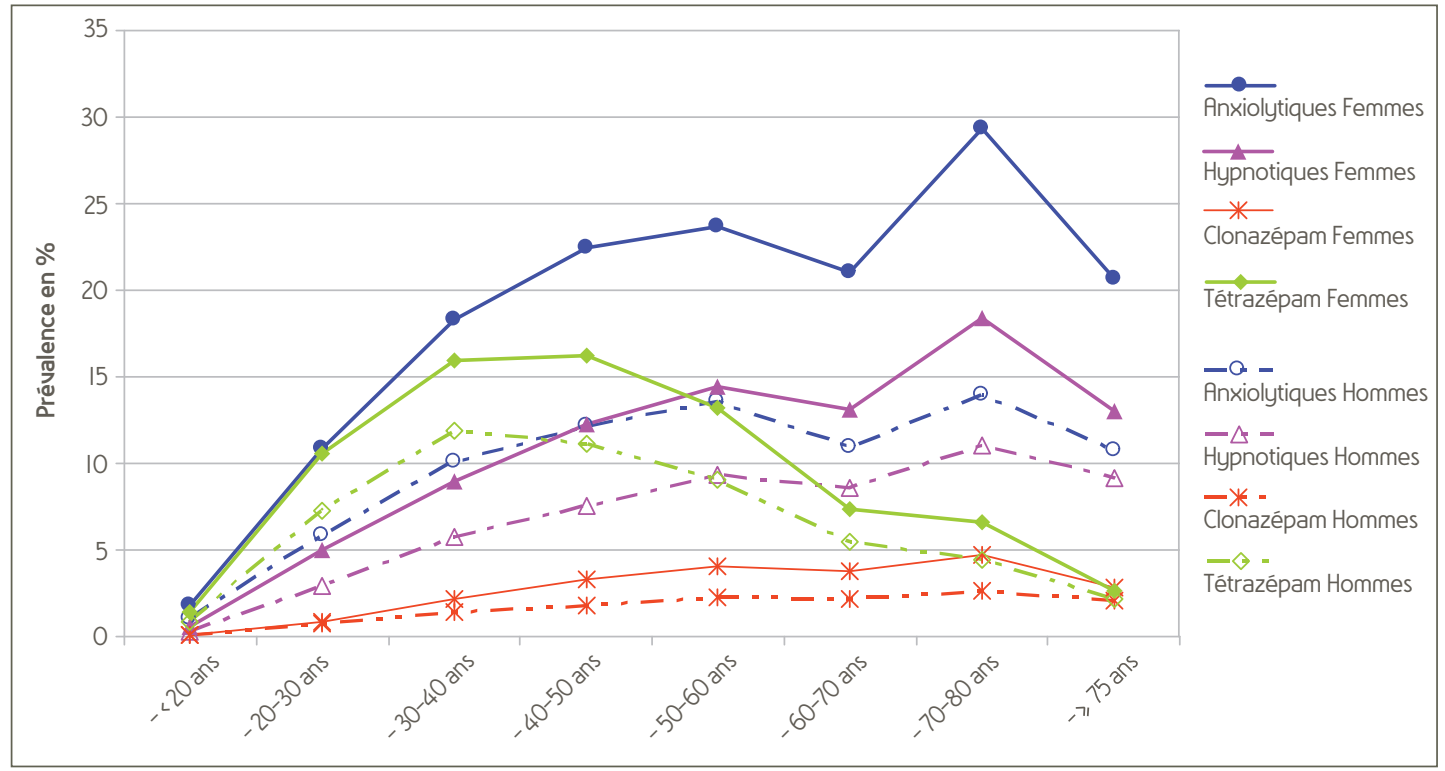

* Nombre de sujets sous BZD extrapolé rapporté à la population française en 2010 (données InSEE)

Les sujets consommant des benzodiazépines sont majoritairement des femmes (près de $60 \%$ ). La consommation des anxiolytiques et des hypnotiques augmente avec l'âge. Les femmes les plus jeunes consommant des benzodiazépines consomment principalement des anxiolytiques et le tétrazépam, les plus âgées des anxiolytiques et des hypnotiques. Les hommes les plus jeunes consomment préférentiellement le tétrazépam, les plus âgés les anxiolytiques et hypnotiques.

\subsection{Co-morbiditēs et traitements associēs}

La présence d'une des 30 affections longue durée, prises en charge à $100 \%$ par l'assurance maladie (ALD30) chez des patients traités par benzodiazépine au cours du traitement ou 12 mois avant, a été collectée. Ces affections sont principalement les troubles mentaux et du comportement, les cancers, certaines maladies rhumatologiques chroniques, les maladies neurologiques, hypertension et le diabète.

Parmi les patients de cette cohorte, la présence d'au moins un remboursement pour un médicament antidépresseur (classe ATC 'n06A') et antipsychotique (classe ATC 'n05A') a été collectée au cours d'un traitement par benzodizépine.

2। \% des patients ont une association de 2 benzodiazépines au cours du traitement. $30 \%$ des patients ont deux benzodiazépines associées, anxiolytiques et/ou hypnotiques. Le clonazépam n'est associé à une autre benzodiazépine ou apparentée que dans $18 \%$ des cas et le tétrazépam que dans 7 \% (Annexe III, Table All). Un antidépresseur est associé à une benzodiazépine dans 21,I \% et à un traitement antipsychotique dans $4,9 \%$ des cas. 
Parmi les patients traités par benzodiazépines ou apparentées, $24,2 \%$ ont au moins une ALD30. Cette proportion est supérieure à celle de la population affiliée au régime général publiée par la CnAmTS(6) en 2006 (environ II à $19 \%$ pour les bénéficiaires âgés de 30 à 60 ans en 2006) [7].

Cette proportion est variable en fonction de l'indication de la benzodiazépine prescrite: près de $35 \%$ des malades sous une benzodiazépine anxiolytique ou clonazépam bénéficient d'une ALD30, ainsi que $29 \%$ des patients traités par une benzodiazépine hypnotique. Par contre, seulement II \% des patients sous tétrazépam bénéficient d'une ALD30

Les principales pathologies retrouvées dans cette population sont majoritairement un cancer (6,0\%), un diabète $(5,0 \%)$ et des troubles psychiatriques (4,9\%). À noter que la proportion d'ALD30 attribuée pour une pathologie d'origine rhumatologique est la plus importante chez les sujets traités par clonazépam (3,1 \%) et non par tétrazépam (0,9 \%) (Annexe III, Table All).

En moyenne, un patient sur 4 traités par une benzodiazépine est atteint d'une pathologie chronique. Cependant, cette proportion est plus élevée pour les patients traités par des anxiolytiques $(34,5 \%)$ ou par clonazépam (38,4\%) et est plus faible pour les patients traités par tétrazépam (II,4 \%).

\subsection{Modalités de traitement par une benzodiazépine}

\section{a. Qui sont les prescripteurs de benzodiazépines ?}

Les données disponibles concernent le mode d'exercice (libéral ou hospitalier) du prescripteur, sill est médecin traitant ou non (si exercice libéral) et la spécialité médicale qu'il exerce (si libérall. Celle-ci nést pas ou peu renseignée pour les praticiens exerçant dans un établissement de soins. Les dates et nombre de consultations médicales permettent le suivi médical du patient au cours du temps. Le nomadisme médical peut être obtenu à partir du nombre de prescripteurs différents consultés au cours du traitement pour se procurer le médicament (plus de 4 professionnels).

Les primo-prescriptions de BZD, toutes classes confondues sont établies par des médecins libéraux dans $88 \%$ des cas

Ces prescripteurs libéraux sont des médecins généralistes dans la presque totalité des cas (plus de $90 \%$ ), sauf pour le clonazépam qui est prescrit à $81 \%$ par des médecins généralistes mais également par des rhumatologues. Les psychiatres prescrivent le plus, après les généralistes, les anxiolytiques (3,7\%) et les hypnotiques (2,3\%), puis ce sont ensuite les rhumatologues pour le tétrazépam (2\%) (Annexe III, Table AIII).

Ce sont les benzodiazépines hypnotiques qui donnent lieu au nombre le plus élevé de consultations $(8,9)$ pendant le traitement, et le tétrazépam au nombre le plus faible de consultations (I,5) (Annexe III, Table fIV). La proportion de patients bénéficiant d'une seule consultation pour se procurer une benzodiazépine est de $54,6 \%$ pour une benzodiazépine anxiolytique, 51,3\% pour une benzodiazépine hypnotique, $59,3 \%$ pour le clonazépam et $82,3 \%$ pour le tétrazépam. Parallèlement, $28,3 \%$ des patients se procurant une benzodiazépine anxiolytique consultent un médecin plus de 3 fois. Cette proportion est de 31,l \% lorsqu'il s'agit d'une benzodiazépine hypnotique, 19,7\% pour le clonazépam et 3,8\% pour le tétrazépam.

Pour $5 \%$ des prescriptions d'hypnotiques et d'anxiolytiques, il existe un nomadisme médical, c'est-à-dire que le patient va consulter plus de 4 prescripteurs différents au cours du traitement (Annexe III, Table AIV). Ce chiffre est plus faible pour les prescriptions de clonazépam (2,7\%) et de tétrazépam (moins de I \%).

(6) Caisse Mationale d'Assurance Maladie. 


\section{b. Indicateurs liés à la délivrance de benzodiazépines en cours de traitement}

Comme pour les données concernant les consultations médicales, les dates de remboursement $1=$ dates de délivrance) des médicaments sont disponibles à partir de l'échantillon des bénéficiaires. Par différence, le délai moyen entre 2 délivrances est ainsi estimé pour chaque patient.

Les quantités déliurées sont calculées à chaque remboursement à partir du nombre de boîtes de médicament remboursé (délivré). La dose moyenne journalière est estimée en rapportant la quantité totale cumulée à la durée de traitement. La durée de traitement correspond au temps écoulé entre la date de lre délivrance du médicament et la date considérée comme la dernière date de délivrance, c'est-à-dire lorsquill n'y a plus de délivrances pendant au moins 3 mois. Lorsquiune nouvelle délivrance est honorée dans un délai de plus de 3 mois, le patient est considéré comme débutant un nouveau traitement ( 2 « cycle »). Les posologies moyennes et durée d'exposition ont été déterminées uniquement à partir des données du premier cycle de traitement rencontré par bénéficiaire.

62,5\% des patients ne bénéficient que d'une seule déliurance de benzodiazépines (premier cycle de traitement), toutes catégories confondues : environ la moitié des patients sous benzodiazépine hypnotique (ou apparentée) ou anxiolytique ne bénéficie que d'une seule déliurance (5l et $52 \%$ respectivement). Ce chiffre est un peu plus élevé pour le clonazépam (55\%). Pour le tétrazépam, 82 \% des patients traités ne bénéficient que d'une seule délivrance (Annexe III, Table AV). Quelle que soit l'indication de la benzodiazépine, la tranche d'âge ne bénéficiant que d'une seule délivrance est majoritairement celle des 30-50 ans. En effet, plus de $30 \%$ (31 \% pour le clonazépam, 35 \% pour une benzodiazépine hypnotique, 40 \% pour une benzodiazépine anxiolytique et $47 \%$ pour le tétrazépam) des patients de cet âge ne renouvelle pas leur traitement dans les 3 mois qui suivent. La proportion de patients ne bénéficiant que d'une seule délivrance est minimale lorsqu'ills sont âgés de plus de 70 ans lentre 5 et $21 \%$ selon l'indication, Annexe III, Table AV).

II y a environ 9 déliurances pour un traitement continu (premier « cycle » de traitement depuis le ler juillet 2006) de benzodiazépines anxiolytiques et hypnotiques ou apparentées. Pour le clonazépam, il n’y a que 5 déliurances et 2 pour le tétrazépam.

Le délai médian entre 2 déliurances chez les patients bénéficiant de plus de 2 remboursements est peu différent selon l'indication et est proche de I mois ${ }^{(7)}$.

Le temps de traitement médian est de 7 mois pour une benzodiazépine anxiolytique et hypnotique ou apparentée, de 4 mois pour le clonazépam et 2 mois pour le tétrazépam (Annexe III, Table AV).

Ce sont les sujets les plus âgés qui consomment les benzodiazépines et apparentées pour une durée de traitement de plus de 3 mois. Lorsque les patients ont plus de 50 ans, $80 \%$ d'entre eux sont traités plus de 3 mois par une benzodiazépine anxiolytique et hypnotique, $70 \%$ par le clonazépam et $50 \%$ par le tétrazépam (Annexe III, Table AU).

Environ $18 \%$ des patients ont une posologie journalière estimée supérieure à celle recommandée par l'autorisation de mise sur le marché (Amm). La posologie de l'Amm est respectée dans $84 \%$ des cas quand il s'agit d'une benzodiazépine hypnotique. Elle ne l'est que dans 76 \% quand il s'agit du tétrazépam (Annexe III, Table AU). Les benzodiazépines dont la dose journalière estimée dépasse la dose maximale le plus fortement sont: le loflazépate d'éthyle (47,8\%), l'estazolam (45,3\%), le nitrazépam (42,9\%), le zolpidem (27,5\%) et le lormétazépam (25,2\%).

(7) IQR: 0,9-I,7. 


\section{c. Suivi des patients sous benzodiazépines et apparentées pendant 5 ans}

Chaque patient peut être exposé plusieurs fois à un traitement de benzodiazépines ou apparentées au cours des 5 ans de la période étudiée. Un « cycle » de traitement par benzodiazépines est défini lorsquiil existe une succession de délivrances pharmaceutiques dont le délai entre 2 délivrances (pour le même médicament) est de moins de 3 mois. Toute délivrance, au-delà de 3 mois, de la même benzodiazépine ou d'une autre, est considérée comme une initiation d'un nouveau cycle de traitement. Chaque cycle a été identifié pour chaque patient.

Parmi les patients sélectionnés, le nombre moyen de « cycles » de traitement par benzodiazépines ou apparentées au cours de ce suivi est de 2,4t|,7 par patient. $53 \%$ des patients auront au moins un nouveau cycle de traitement au cours des 5 années de suivi et $5 \%$ d'entre eux plus de 6 cycles de traitement, de durée très variable. Au cours de ces 5 années, le nombre de benzodiazépines différentes auxquelles est

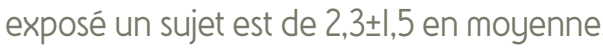

Au cours des cinq années de suivi, avec ou sans interruption de traitement (i.e. prise en compte de toutes les déliurances sur cette période, quel que soit le délai entre 2 déliurances), 25 à $30 \%$ des sujets traités sont exposés moins de 3 mois à une benzodiazépine anxiolytique et hypnotique, 33 \% au clonazépam et $50 \%$ au tétrazépam (Annexe III, Table AVI).

Par ailleurs, près de $52 \%$ des sujets traités sont exposés plus de 2 ans à une benzodiazépine anxiolytique et hypnotique, $46 \%$ pour le clonazépam et $30 \%$ pour le tétrazépam, même s'ils ne sont pas traités de manière continue (Annexe III, Table AVII).

La probabilité qu'un patient reprenne une nouvelle benzodiazépine à 2 ans est de 60 \% lorsque la benzodiazépine est anxiolytique, hypnotique ou le clonazépam, mais elle est nettement diminuée à $43 \%$ lorsque la benzodiazépine est le tétrazépam (Annexe III, Table AVIII).

Plus de la moitié ( $55 \%$ ) des patients ayant bénéficié d'un arrêt de traitement de benzodiazépines reprendra au moins une fois un nouveau traitement dans les 24 mois qui suivent, avec un délai de survenue médian proche de 21 mois. 


\section{Les risques liés à l'usage des benzodiazépines}

\section{I. Les risques}

\section{I.I. Les risques généraux}

L'ensemble des risques généraux liés à l'usage des benzodiazépines est bien connu et figure dans le Résumé des Caractéristiques du Produit (Amm) de chacune d'elle [8]. Ainsi, l'usage des benzodiazépines peut entraîner:

- une amnésie antérograde (perte de la mémoire des faits récents), qui peut survenir aux doses thérapeutiques. Le risque augmente proportionnellement à la dose;

- une altération des fonctions psychomotrices pouvant survenir dans les heures suivant la prise;

- un syndrome associant, à des degrés divers, des troubles du comportement et de la mémoire et une altération de l'état de conscience. Peuvent être ainsi observés les effets suivants: aggravation de l'insomnie, cauchemars, agitation, nervosité, idées délirantes, hallucinations, état confuso-onirique, symptômes de type psychotique, désinhibition avec impulsivité, euphorie, irritabilité, amnésie antérograde et suggestibilité.

Ce syndrome peut s'accompagner de troubles potentiellement dangereux pour autrui et pour lui-même tel qu'un comportement inhabituel pour le patient, un comportement violent, notamment si l'entourage tente d'entraver l'activité du patient

Ces manifestations imposent l'arrêt du traitement [8]. En cas d'impossibilité d'arrêt complet du traitement, une diminution de la posologie peut, dans certains cas, faire régresser les troubles.

- une tolérance caractérisée par une diminution progressive de l'effet thérapeutique pour une même dose administrée pendant plusieurs semaines. La tolérance peut conduire à une augmentation des doses pour obtenir l'effet recherché;

- une dépendance. Tout traitement par les benzodiazépines et apparentées, et plus particulièrement en cas d'utilisation prolongée, peut entraîner un état de pharmacodépendance physique et psychique. Divers facteurs semblent favoriser la survenue de la dépendance : la durée du traitement, la dose et les antécédents d'autres dépendances médicamenteuses ou non, y compris alcoolique.

Une pharmacodépendance peut survenir à doses thérapeutiques et/ou chez des patients sans facteur de risque particulier.

L'association de plusieurs benzodiazépines risque, quelle qu'en soit l'indication, d'accroître le risque de pharmacodépendance [8].

L'arrêt du traitement par une benzodiazépine, même prise à posologie normale, peut entraîner un phénomène de sevrage. Peuvent alors être observés des céphalées, douleurs et faiblesse musculaires, cauchemars, irritabilité, agitation, tremblements, anorexie, nausées, sueurs, diarrhée. Plus sévèrement: convulsions, changements d'humeur, dépression, dépersonnalisation, désorientation, hallucinations, psychose paranoïde, Le syndrome de sevrage peut se manifester dans les jours qui suivent l'arrêt du traitement. Pour les benzodiazépines à durée d'action brève, et surtout si elles sont données à doses élevées, les symptômes peuvent même se manifester dans l'intervalle qui sépare deux prises.

Le syndrome de seurage est à distinguer du phénomène de rebond, transitoire, caractérisé par une exacerbation du symptôme ayant motivé le traitement par une benzodiazépine ou apparentée (rebond d'anxiété ou d'insomnie). 


\section{I.2. Les associations avec d'autres médicaments ou d'autres substances}

L'alcool majore l'effet sédatif des benzodiazépines et apparentées.

L'association avec d'autres dépresseurs du système nerveux central augmente la dépression centrale et l'altération de la vigilance. L'association avec les dérivés morphiniques lanalgésiques, antitussifs et traitement de substitution de la pharmacodépendance aux opiacés) majore également le risque de dépression respiratoire, pouvant être mortelle.

\section{5.l.3. Les risques liếs à une utilisation dans des situations particulières \\ - Benzodiazépines et sujet âgé}

La consommation de benzodiazépines expose les sujets âgés à des risques spécifiques en raison des modifications physiologiques liées à l'âge. Ces modifications favorisent une accumulation des métabolites actifs des benzodiazépines et accroissent ainsi le risque de surdosage et d'effets indésirables.

Ces effets indésirables sont essentiellement psychomoteurs et cognitifs, avec l'augmentation des risques de chutes et de fractures, la perturbation de la mémoire à court terme, de la mémoire de rappel, un ralentissement dans l'apprentissage d'une nouvelle information, des performances verbales ainsi qu'un risque de déclin cognitif [9-13].

\section{- Benzodiazépines et démence}

L'Afssaps examine actuellement le lien entre benzodiazépines, démences dont la maladie d'Alzheimer suggéré par plusieurs études. [14-22]

Les troubles de la mémoire ont un effet indésirable bien connu des traitements par benzodiazépines. Plusieurs études ont tenté d'évaluer le risque d'altération de la fonction cognitive voire d'apparition d'une démence et son association à l'exposition aux benzodiazépines. Dix études publiées ont été retenues et les résultats ne sont pas concordants. Cinq études concluent à une association, une étude retrouve un effet protecteur des benzodiazépines sur l'apparition d'une démence et quatre ne retrouvent pas d'association ou ne permettent pas de la mettre en évidence pour des raisons statistiques. Le tableau ci-dessous répertorie ces études.

\begin{tabular}{|c|c|c|}
\hline Auteur & Journal & Date \\
\hline \multicolumn{3}{|l|}{ Association positive } \\
\hline Wu et al. (Taïwan) [14] & Am J of Ceriatric Psychiatry & 2010 \\
\hline Uu et al. (Taïwan) [15] & Am J of Ceriatric Psychiatry & 2009 \\
\hline Lagnaoui et al. (France) [16] & J of Clin Epidem & 2002 \\
\hline Paterniti et al. (France) [17] & J of Clin Psychopharm & 2002 \\
\hline Gallacher et al (Royaume Uni) [l8] & J Epidemiol Community Health & 2011 \\
\hline \multicolumn{3}{|l|}{ Pas d'association } \\
\hline Lagnaoui et al. (France) [19] & Age and ageing & 2009 \\
\hline Allard et al. (France) [20] & Intern J of Ceriatric Psy & 2003 \\
\hline Hanlon et al. (USA) [2I] & Clin Pharmacol and Therap & 1998 \\
\hline Dealberto et al. (USA) [22] & Intern J of Ceriatric Psy & 1997 \\
\hline \multicolumn{3}{|l|}{ Association protectrice } \\
\hline Fastbom et al. (Suède) [23] & Alzheimer disease and associated disorders & 1998 \\
\hline
\end{tabular}


En France, le sujet fait l'objet d'une préoccupation particulière du fait d'une consommation importante de benzodiazépines dans la population et d'une consommation dont la durée excède souvent celle des Amm ou des recommandations. Elle s'inscrit également dans le cadre du Plan Alzheimer qui est une priorité de santé publique depuis 4 ans. Enfin, le sujet a fait l'objet d'une médiatisation importante lors de la parution récente d'un article mettant en avant les résultats d'une étude qui établirait un lien entre benzodiazépine et démence. Suite à cette médiatisation, l'équipe ayant mis en place cette étude a communiqué à l'Afssaps une note faisant le point sur ces travaux dont elle a précisé que les résultats n'étaient pas définitifs.

L'ensemble des études a été analysé par le groupe PCR-PEP|(8). Ses membres ont souligné la difficulté à mener des études aux conclusions définitives sur cette question. On peut ainsi citer la difficulté de s'assurer que les sujets inclus dans une étude ne présentaient pas déjà des premiers symptômes de démence avant la mise sous traitement, ces symptômes ayant même pu être à l'origine de la prescription de benzodiazépines (ex: insomnie, anxiété). C'est ce que l'on appelle le biais protopathique ${ }^{(9)}$. De plus, le recueil exhaustif des données sur l'exposition totale aux benzodiazépines (dose, durée) peut être difficile à obtenir. Enfin, la difficulté de recueil et donc prise en compte des facteurs de confusion potentiels peut être soulignée.

Le caractère hétérogène voire discordant des résultats des études retenues reflète les limites méthodologiques des études menées. Les principales sont:

- le biais protopathique;

- le biais de déplétion des susceptibles ${ }^{(10)}$;

- l'absence d'information sur la durée réelle de l'exposition aux benzodiazépines (dose, posologie, durée de traitement et donc dose cumulée) qui peut être à l'origine d'un biais de classement;

- l'exclusion de nombreux sujets des cohortes initiales pouvant être à l'origine de biais de sélection si la raison d'exclusion est liée à la démence lex: inclusion uniquement de sujets aptes à répondre aux questions) ou de biais de classement (ex: inclusion uniquement de sujets pour lesquels l'information sur l'utilisation de benzodiazépines est disponible entre l'inclusion dans l'étude et la date de survenue de la démence);

- les différences dans le mode de recueil des données entre les études;

- la représentativité de certains échantillons et donc l'extrapolation des résultats;

- la non prise en compte de manière satisfaisante de certains facteurs de confusion potentiels;

- les modalités de prise en compte de l'effet de l'âge qui est un facteur important de la maladie d'Alzheimer.

Le groupe a proposé d'analyser les résultats de l'étude citée ci-dessus lorsque ceux-ci seront disponibles. Il a également été proposé de solliciter d'autres cohortes de suivi de sujets âgés dont un des objectif est d'étudier les facteurs de risque de démence pour connaître leurs données sur cette association benzodiazépines/démence ou la faisabilité d'une recherche dans ce domaine:

(8) Groupe PCR-PEPI : groupe Plan de gestion de risque - Etudes pharmacoépidémiologiques

(9) Dictionnaire de pharmaco-épidémiologie - B Bégaud: « distorsion de l'estimation de l'association entre la prise d'un médicament et la survenue d'un effet indésirable liée au fait que le début de l'exposition à un facteur de risque (par exemple, un traitement médicamenteux) est, en fait, postérieur à la survenue de l'événement ou tout au moins à celle de ses premières manifestations. Dans une forme plus restrictive (biais lié à la maladie, disease confounding), la prise ou la prescription du médicament peut même être motivée par l'apparition des premiers symptômes de la maladie. »

(IO) Dictionnaire de pharmaco-épidémiologie - B Bégaud: « disparition, plus ou moins rapide, au cours du suivi d'une population, du sous-groupe des sujets à risque élevé si ceux-ci cessent d'être suivis ou ne sont plus à risque après avoir présenté l'événement indésirable. » 
- cohorte française dite des 3 cités pour laquelle environ 10000 personnes sont suivies pendant 10 ans dans trois villes françaises, Bordeaux, Dijon et Montpellier afin d'identifier les facteurs de risque et de protection de la maladie d'Alzheimer;

- cohorte internationale en Italie, aux Pays-Bas et aux États-Unis.

Le groupe PCR-PEPI a considéré, qu'à l'heure actuelle, les données disponibles ne permettent pas de conclure à l'existence ou non d'une association entre benzodiazépines et démence du fait des limites mentionnées ci-dessus.

L'importance de ce sujet mérite une nouvelle prise en compte de ce risque à court terme. Ainsi, il a été convenu que les travaux actuellement en cours feront l'objet d'une nouvelle analyse lors de la parution des résultats de cette dernière étude.

\section{- Benzodiazépines et conduite automobile}

Le lien entre la consommation de benzodiazépines et la survenue d'accidents de la route a été largement investigué lors de diverses études internationales [24]. L'augmentation du risque d'accidents par les benzodiazépines est démontrée aussi bien chez le sujet âgé que chez des conducteurs plus jeunes. En France, un pictogramme indiquant le niveau de risque (classification à trois niveaux) est apposé sur le conditionnement externe des médicaments susceptibles « d’altérer les capacités à conduire un véhicule ». Tous les médicaments de la famille des benzodiazépines relèvent des niveaux de risque les plus élevés de cette classification mise en œuure par l'Afssaps (niveaux 2 et 3 ; cf. annexe V).

La pertinence de ce classement a été confirmée, lors d'une importante étude pharmaco-épidémiologique soutenue par l'Afssaps [25-27], mettant en regard les données recueillies par les forces de l'ordre sur les accidents de la circulation avec celles de remboursement des médicaments de l'Assurance Maladie (SnIIIRAM). À partir d'un échantillon de 3843 conducteurs exposés aux benzodiazépines et apparentées, il a été mis

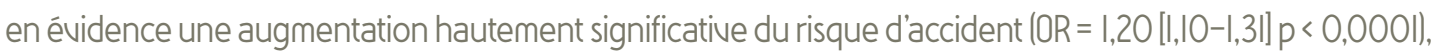
après ajustement sur le sexe, la catégorie socioprofessionnelle, la classe d'âge et d'autres facteurs de risque (heure et localisation de l'accident, type de véhicule, gravité des blessures, alcool, prise d'autres médicaments potentiellement dangereux.... De plus, cette étude comparant les conducteurs responsables aux conducteurs non-responsables, a permis d'estimer à l,03 \% la part attribuable aux benzodiazépines de l'ensemble des accidents de la route survenant en France.

\section{- Benzodiazépines en milieu professionnel}

En 2006, une étude réalisée sur des salariés français a montré que, sur une population de 2213 salariês enquêtés, 6 \% prenaient un médicament psychoactif. Le nombre de femmes était plus élevé. La majorité avait recours à un anxiolytique ou un hypnotique [28,29]. Les facteurs favorisant l'initiation d'un médicament psychotrope sont différents selon le sexe : situation matrimoniale (divorce,...) et catégorie socioprofessionnelle chez les hommes; âge et conditions de travail chez les femmes (plus de contraintes professionnelles rapportées par les femmes par rapport aux hommes : travail sans créativité, absence de prise de décision, tensions). Il a également été montré qu'une consommation de médicaments psychoactifs à long terme avait un impact négatif sur les fonctions cognitives notamment avec une altération de la mémoire en cas d'usage chronique d'anxiolytiques et une altération de l'attention sélective en cas d'usage d'hypnotiques au long cours chez les femmes [30,31]. 


\section{I.4. L'usage problématique}

Les benzodiazépines et apparentées font l'objet d'une surveillance active par les Centres d'Évaluation et d'Information sur la Pharmacodépendance (CEIP), réseau d'addictovigilance de l'Afssaps. L'évaluation des données recueillies a permis de mettre en évidence 3 types de risques:

- le risque d’abus, de dépendance et d'usage détourné;

- le risque d'usage criminel, en particulier de soumission chimique;

- le risque de prescription hors-Amm

\section{- Le risque de dépendance, d'abus et d'usage détourné}

Le réseau d'addictovigilance a mis en évidence un abus et un usage détourné de certaines benzodiazépines par les usagers de drogues.

En 20I0, les 5 benzodiazépines faisant le plus l'objet d'abus par les patients suivis par les médecins généralistes sont le zolpidem, le bromazépam, l'oxazépam, la zopiclone et l'alprazolam (enquête OPEMA (II) 20I0). Les 5 benzodiazépines les plus fréquemment consommées par les patients suivis dans des structures spécialisées dans les soins aux toxicomanes sont l'oxazépam, le diazépam, le clonazépam, le bromazépam et la zopiclone. Cependant, le flunitrazépam et le clonazépam sont les molécules qui ont les indicateurs de détournement les plus élevés. Les indicateurs de détournement sont la souffrance à l'arrêt de la substance, les cas d'abus et de dépendance, l'obtention illégale et une dose consommée supérieure à deux fois celle recommandée dans l'Amm (enquête OPPIDUIm (12) 20I0).

Par ailleurs, les médicaments contenant zolpidem, clonazépam, bromazépam, flunitrazépam, zopiclone et alprazolam figurent parmi les plus retrouvés sur les ordonnances suspectes de falsification présentées aux pharmaciens d'officine (enquête OSIAP ${ }^{(13)}$ 20I0)

\section{- Le risque d'usage criminel, en particulier de soumission chimique}

La soumission chimique se définit comme l'administration à des fins criminelles (viols, actes de pédophilie) ou délictuelles (violences volontaires, vols) de substances psychoactives à l'insu de la victime ou sous la menace. En 2003, une enquête annuelle a été mise en place par l'Afssaps pour identifier les substances et les médicaments les plus souvent en cause.

Ce sont les benzodiazépines qui sont les substances les plus fréquemment impliquées dans les cas de soumission chimique. Dans les résultats de l'enquête 20I0, le clonazépam, la zopiclone et le zolpidem, sont les molécules les plus souvent retrouvées.

\section{- Le risque de prescription hors-Amm}

Les outils de surveillance de l'utilisation des médicaments ont mis en évidence l'existence des prescriptions hors Amm pour certaines benzodiazépines. C'est en particulier le cas du clonazépam, indiqué dans le traitement de l'épilepsie mais majoritairement prescrit dans d'autres indications, notamment la douleur, les troubles du sommeil et les troubles anxieux.

(II) OPEMA : Observatoire des Pharmacodépendances en médecine ambulatoire

(I2) OPPIDUM : Observations des psychotropes et produits illicites Détournés de leur Usage médicamenteux

(I3) OSIAP: Ordonnances Suspectes, Indicateur d'Abus Possible 


\section{Les actions des autorités sanitaires}

\section{I. Les actions réalisées}

Toutes ces actions sont détaillées dans l'annexe V.

\section{I.I. Amélioration de l'information des professionnels de santé et des patients}

Elle porte sur 4 principaux types d'action:

- l'harmonisation du résumé des caractéristiques du produit (RCP) et de la notice des benzodiazépines: depuis 2004, un schéma commun d'informations figure dans les RCP et notices de l'ensemble des benzodiazépines et apparentées. Le risque de pharmacodépendance est mentionné pour toutes les spécialités;

- la diffusion de communiqués et de lettres d'information aux professionnels de santé:

peuvent être cités, à titre d'exemple, le point d'information sur le Rohypnol ${ }^{\circledR}$ (flunitrazépam) [32], l'information sur le bon usage du Rivotri| ${ }^{\circledR}$ (clonazepam) [33], l'information sur les modifications des conditions de prescription et de déliurance du Rivotril) [34];

- dans le cadre de l'amélioration de la qualité des soins, l'Assurance Maladie a conçu récemment le programme d'évolution des pratiques intitulé CAPI destiné aux médecins traitants afin de les accompagner dans leur démarche d'amélioration des soins. Afin de lutter contre la iatrogénie médicamenteuse chez les plus de 65 ans, il leur est ainsi recommandé de diminuer le recours aux benzodiazépines à longue demi-vie dans cette population.

- l'élaboration et la publication de mises au point et de recommandations

peuvent être cités, à titre d'exemple, la mise au point sur les troubles du comportement liés à l'utilisation des benzodiazépines et produits apparentés [35]; le programme « Améliorer la prescription des psychotropes chez le sujet âgé », [36] la mise au point sur l'arrêt des hypnotiques, [37] la mise au point sur l'arrêt de l'utilisation hors Amm du clonazépam (Rivotril ${ }^{\circledR}$ ) [32];

- pictogramme sur les conditionnements externes des médicaments.

En 2005, a été instaurée une classification à trois niveaux du pictogramme apposé sur le conditionnement externe des médicaments susceptibles « d'altérer les capacités à conduire un véhicule et, par extension, à utiliser des machines potentiellement dangereuses ou à exécuter des travaux nécessitant une attention soutenue ». Les médicaments de la famille des benzodiazépines relèvent tous des niveaux de risque les plus élevés (niveaux 2 et 3; cf. Annexe V).

\section{I.2. Encadrement de la prescription et de la déliurance}

Depuis 1991, la durée maximale de prescription est limitée à 12 semaines pour les anxiolytiques et 4 semaines pour les hypnotiques.

Elle a été réduite de façon plus importante pour certains médicaments hypnotiques (par exemple limitation de la durée de prescription du flunitrazépam à 14 jours en 200I).

De plus, la restriction de la durée maximale de prescription a également été un levier pour limiter l'abus du clonazépam (limitation à 12 semaines depuis 20I0). 
Pour certaines benzodiazépines particulièrement détournées notamment par les toxicomanes, la prescription sur ordonnance sécurisée est devenue obligatoire (flunitrazépam, clorazépate dipotassique et clonazépam).

Par ailleurs, les indications de certaines benzodiazépines ont été restreintes (par exemple, flunitrazépam en 1996, cf. annexe V). La prescription du clonazépam va également être restreinte à certains prescripteurs

\section{6.l.3. Prévention du risque de soumission chimique et d'abus}

Certaines modifications de la galénique ont été apportées aux benzodiazépines pour limiter le risque de soumission chimique lajout d'un colorant dans les comprimés de Rohypno ${ }^{\circledR}$, modification en cours pour d'autres benzodiazépines).

La taille des conditionnements a été réduite pour limiter le risque d’abus (flunitrazépam, clonazépam).

\section{I.4. Surveillance sanitaire}

L'ensemble des benzodiazépines fait l'objet d'une surveillance par les réseaux de pharmacovigilance et d’addictovigilance. Les benzodiazépines les plus impliquées dans les cas d'abus et d'usage détourné font l'objet d'une surveillance renforcée et continue par l'Afssaps et son réseau d'addictovigilance. Un plan de gestion de risque a également été mis en place pour les benzodiazépines les plus problématiques (Rivotril ${ }^{\otimes}$ ).

\subsection{Les pistes d'amélioration}

Les différentes données présentées dans ce rapport indiquent clairement que les mesures qui ont déjà été mises en place par les autorités sanitaires ont permis de stabiliser voire de diminuer la consommation des benzodiazépines et de favoriser leur bon usage. Il est ainsi nécessaire de poursuivre dans cette voie et de trouver de nouvelles pistes afin d'optimiser leur usage, limiter les risques liés à leur consommation et diminuer leur surconsommation.

Des actions de différents types peuvent être envisagées mais la communication vers les professionnels de santé et en particulier les médecins généralistes, principaux prescripteurs de benzodiazépines, est une priorité. Certains messages essentiels deuront être rappelés comme, par exemple, la nécessité de « peser » la première prescription, de limiter les posologies, de limiter la prescription dans le temps, de ne pas associer plusieurs benzodiazépines entre elles et de réévaluer la pertinence du traitement régulièrement.

Les thérapeutiques non médicamenteuses comme la psychothérapie sont des alternatives qui doivent aussi être prise en compte. II semble également important de renforcer la formation initiale et continue des médecins sur les psychotropes.

Parallèlement, l'information vers le grand public doit être développée afin de l'avertir que les benzodiazépines ne sont pas des médicaments anodins. En effet, même si elles restent indispensables dans l'arsenal thérapeutique, leurs effets secondaires à court terme et les possibles effets secondaires à long terme invitent à leur utilisation prudente et raisonnée. 
Des mesures d'ordre réglementaire peuvent également être envisagées pour favoriser le bon usage des benzodiazépines comme l'extension de la prescription de l'ensemble des benzodiazépines sur ordonnance sécurisée. Celle-ci est, en effet, une mesure efficace qui permet notamment de limiter le détournement des médicaments et de renforcer l'acte de prescription.

Concernant cette problématique, l'Afssaps a mis en place une enquête visant à identifier les avantages et les inconvénients de la prescription des médicaments sur ordonnance sécurisée et d'évaluer leur acceptabilité auprès des prescripteurs. Les résultats de cette enquête, qui seront connus dans le courant 2012, permettront le cas échéant d'étendre cette prescription à d'autres médicaments que les stupéfiants et pourrait ainsi être envisagé pour la classe des benzodiazépines.

Une autre piste intéressante est la réduction de la taille des conditionnements des benzodiazépines. Les conditionnements actuels ne sont pas tous adaptés à une durée de prescription courte, ni même à la durée maximale de prescription. Cette mesure permettra de mieux adapter la quantité déliurée à la quantité prescrite. Elle permettra également de réduire le risque d’abus.

Des études sur les déterminants sociaux, économiques et culturels liés au recours aux benzodiazépines et aux médicaments psychotropes permettraient de mieux comprendre les raisons de la forte consommation de psychotropes en France et ainsi d'adapter les actions mises en œuure par les autorités sanitaires.

L'impact de l'ensemble des mesures mises en place par les autorités sanitaires continuera à être évalué par les différents réseaux de surveillance de l'Agence et par les données de pharmaco-épidémiologie. L'étude et l'analyse des bases des données de l'Assurance maladie seront également absolument nécessaires au suivi de ces mesures et à leur impact sur la consommation des benzodiazépines.

L'étude des éventuels reports de prescription vers d'autres médicaments sera également indispensable.

L'Agence prévoit ainsi d'organiser courant 2012 une réunion scientifique visant à débattre des mesures les plus adaptées pour limiter les risques liés à la consommation de benzodiazépines et à proposer des études permettant d'étudier et prendre en compte le risque potentiel de démence, dont la maladie d'Alzheimer, associé à la consommation de benzodiazépines 


\section{Références bibliographiques}

I. Legrain, m., Lecomte, T. Psychotropic drug consumption in France and several European conutries. Bull Acad Matl Med 1997, I81 : 1073-1084.

2. Zarifian, E. Prescription of psychotropic drugs; use, misuse and abuse. Bull Acad natl Med 1998, 182: 1439-1446

3. Lecadet J and al, Médicaments psychotropes: consommation et pratiques de prescription en France métropolitaine. Données nationales 2000. Rev Med Ass Maladie 2003, 34: 75-84

4. Gasquet I. and al. Psychotropic drug use and mental psychiatric disorders in France; results of the general population ESEMeD/MHEDEA 2000 epidemiological study. Encephale 2005, 31 : 195-206

5. Briot $m$., Le bon usage des médicaments psychotropes. Office parlementaire d'évaluation des politiques de santé (OPEPS). 2006.

6. Comparaison des données d'utilisation des médicaments psychoactifs ayant un potentiel de dépendance dans les différents pays d'Europe » de m. Lapeyre-Mestre et A. Palmaro (CEIP de Toulouse). Septembre 20II

7. Weill A. Les bénéficiaires d'ALD au 3I décembre 2006. Point repère n9, septembre 2007. http://www. ameli.fr/fileadmin/user_upload/documents/pt_repere_9.pdf.

8. Résumé - caractéristiques du produit des 22 benzodiazépines

9. Fourrier A, Letenneur L, Dartigues JF, Moore A, Bégaud B. Benzodiazepine use in an elderly communitydwelling population. Characteristics of users and factors associated with subsequent use.Eur J Clin Pharmacol. 200I Aug;57(5):419-25

IO. Zint K, Haefeli UE, Glynn RJ et al. Impact of drug interactions, dosage, and duration of therapy on the risk of hip fracture associated with benzodiazepine use in older adults. Pharmacoepidemiol Drug Saf. 20I0 Dec;19(|2):1248-55.

II. Golden AG, Ma Q, Mair V, Florez HJ, Roos BA. Risk for fractures with centrally acting muscle relaxants: an analysis of a national Medicare Advantage claims database. Ann Pharmacother. 2010 Sep;44(9):1369-75

12. Pariente A, Dartigues JF, Benichou Jet al. Benzodiazepines and injurious falls in community dwelling elders. Drugs Aging. 2008;25(I):61-70

13. Améliorer la prescription des psychotropes chez le sujet âgé. Démarche participative de la HAS. Octobre 2007

14. Uu CS, Ting TT, Wang SC, Chang IS, Lin Km. Effect of Benzodiazepine Discontinuation on Dementia Risk. Am J Geriatr Psychiatry 2010.

15. Wu CS, Wang SC, Chang IS, Lin KM. The association between dementia and longterm use of benzodiazepine in the elderly: nested case-control study using claims data. Am J Geriatr Psychiatry 2009;17(7):614-20.

16. Lagnaoui R, Begaud B, Moore $\mathrm{C}$, Chaslerie A, Fourrier A, Letenneur L, et al. Benzodiazepine use and risk of dementia : a nested case-control study. J Clin Epidemiol 2002;55(3):314-8. 
17. Paterniti S, Dufouil C, Alperovitch A. Long-term benzodiazepine use and cognitive decline in the elderly: the Epidemiology of Vascular Aging Study. J Clin Psychopharmacol 2002;22(3):285-93.

18. Gallacher J, Elwood P, Pickering J, Bayer A, Fish M, Ben-Shlomo Y. Benzodiazepine use and risk of dementia: evidence from the Caerphilly Prospective Study (CaPS). J Epidemiol Community Health. 2OII Oct 27.

19. Lagnaoui R, Tournier M, Moride Y, Wolfson C, Ducruet T, Begaud B, et al. The risk of cognitive impairment in older community-dwelling women after benzodiazepine use. Age Ageing 2009;38(2):226-8.

20. Allard J, Artero S, Ritchie K. Consumption of psychotropic medication in the elderly: a re-evaluation of its effect on cognitive performance. Int J Geriatr Psychiatry 2003;18(I0):874-8.

21. Hanlon JT, Horner RD, Schmader KE, Fillenbaum GG, Lewis IK, Wall WE, Jr., et al. Benzodiazepine use and cognitive function among community-dwelling elderly. Clin Pharmacol Ther 1998;64(6):684-92.

22. Dealberto mJ, McAvay GJ, Seeman T, Berkman L. Psychotropic drug use and cognitive decline among older men and women. Int J Geriatr Psychiatry 1997;2(5):567-74.

23. Fastbom J, Forsell Y, Winblad B. Benzodiazepines may have protective effects against Alzheimer disease. Alzheimer Dis Assoc Disord 1998;2(1):14-7.

24. Orriols L, Salmi LR, Philip et al. The impact of medicinal drugs on traffic safety: a systematic review of epidemiological studies. Pharmacoepidemiol Drug Saf 2009;18(8):647-58.

25. Orriols L, Delorme B, Gadegbeku B et al. Prescribed medicines and the risk of road traffic crashes: results of a French registry-based study. PLoS Med 20I0;7(II): e 1000366.

26. Orriols L, Philip P, moore $\mathrm{n}$ et al. Benzodiazepine-like hypnotics and the related risk of road traffic accidents. Clin PharmTher 2011;89:595-60I.

27. Corsenac P, Lagarde E, Gadegbeku B et al. Road traffic crashes and prescribed methadone and buprenorphine: A french registrybased case-control study. Drug Alcohol Depend 20ll; doi:10.1016/j. drugalcdep.20II.10.022

28. Bœuf-Cazou, 0. et al, Profil de consommation de substances psychoactives dans le milieu du travail: résultats de l'enquête « Mode de Vie et travail », Thérapie 20II, 66: I55-165.

29. Bœuf-Cazou, 0. et al, Evolution of drug consumption in a sample of French workers since 1986: the "Drugs and Work study", Pharmacoepidemiology and drug safety, 2009.

30. Lapeyre-Mestre, m., Bœuf-Cazou, 0., Rapport sur les médicaments et substances psychoactives en milieu du travail: analyse des facteurs de risques et de l'impact d'une exposition chronique, CEIP de Toulouse, 20II, $60 \mathrm{p}$.

31. Miezborala, $\mathrm{m}$. et al, Etude auprès de 2106 travailleurs de la région toulousaine. Communication orale aux journées nationales de médecine et de santé au travail. Lyon, 31 mai 2006.

32. Point d'information concernant le Rohypnol ${ }^{\circledR}$. Afssaps 200 I. http://www.afssaps.fr/Infos-de-securite/ Communiques-Points-presse/Securite- $d$-emploi-des-medicaments-psychotropes-point- $d$ information-sur-le-Rohypnol-flunitrazepam/(language)/fre-FR 
33. Informations importantes sur le bon usage du RIVOTRIL ${ }^{\circledR}$ et sur la réduction du conditionnement des comprimés. Afssaps 2008. http://www.afssaps.fr/content/download/I2807//55809/version/I/file/ ddl-rivotril-072008.pdf

34. Modifications des conditions de prescriptions du Rivotril ${ }^{\circledR}$. Afssaps 20II. http://www.afssaps.fr/var/ afssaps_site/storage/original/application/73d829c70ba66dd5cf6955a3a2l4d458.pdf

35. Sécurité d'emploi des benzodiazépines et produits apparentés. Afssaps 200I. http://www.afssaps.fr/ Infos-de-securite/Communiques-Points-presse/Securite-d-emploi-des-benzodiazepines-et-produitsapparentes/(language)/fre-FR

36. Retrait de l'Amm de noctran ${ }^{\oplus}$ et de Mépronizine ${ }^{\oplus}$ : l'Afssaps émet des recommandations sur la conduite à tenir pour arrêter un traitement par hypnotique - Communiqué. Afssaps 20ll. http://www.afssaps. $\mathrm{fr} /$ Infos-de-securite/Communiques-Points-presse/Retrait-de-I-Amm-de-noctran-R-et-deMepronizine-R-I-Afssaps-emet-des-recommandations-sur-la-conduite-a-tenir-pour-arreter-untraitement-par-hypnotique-Communique/(language)/fre-FR

37. Améliorer la prescription des psychotropes chez le sujet âgé. HAS 2007. http://www.has-sante.fr/ portail/upload/docs/application/pdf/prescription_psychotropes_sujet_age_version_ courte_2007_II_07__19_34_38_576.pdf 


\section{Annexes}

\section{Annexe I: Remarques méthodologiques concernant les sources de données nationales}

\section{Données de ventes des laboratoires}

La plupart des données présentées dans ce recueil ont été converties en nombre de Doses Définies Journalières (DDJ). Établie sous l'égide du « Collaborating Centre for Drug Statistics Methodology » de I'OMS, la Dose Définie Journalière constitue une posologie de référence pour un adulte de soixante-dix kilos dans l'indication principale de chaque molécule. Cette dose moyenne ne reflète pas nécessairement la posologie recommandée par l'Amm ni la posologie effective : elle constitue un étalon de mesure. Chaque présentation d'un médicament peut ainsi être convertie en nombre de DDJ et, si l'on connaît le nombre total de boîtes vendues, la consommation au cours d'une année peut ensuite être calculée, que ce soit par molécule, par famille ou par classe. L'usage des DDJ élimine ainsi les difficultés de mesure liées à l'hétérogénéité des tailles de conditionnement et de dosage des médicaments commercialisés. Pour tenir compte des différences de population d'un pays à l'autre, le nombre de DDJ est divisé par le nombre total d'habitants (enfants compris). Par convention, les résultats sont présentés pour mille habitants et par jour (DDJ// OOOH/J). Cet indicateur rend donc possible les comparaisons de consommations et permet de calculer, le cas échéant, une consommation moyenne internationale. La version de la classification ATC utilisée est celle de janvier 20II. Pour les quelques substances (classées parmi les myorelaxants) ne disposant d'une DDJ officielle fixée par l'OMS, la posologie moyenne mentionnée dans l'autorisation de mise sur le marché a été retenue pour effectuer ces calculs.

Les données utilisées proviennent des déclarations de ventes que les titulaires d'autorisations de mise sur le marché (Amm) adressent chaque année à l'Afssaps. Ces déclarations, prévues à l'article L 5I2I-17 et suivants du Code de la Santé Publique, présentent un caractère obligatoire et portent sur la totalité des spécialités commercialisées en France, qu'elles soient ou non remboursables aux assurés sociaux. Elles sont signalées dans ce rapport par la mention « source: Afssaps ». Les chiffres d'affaires sont exprimés en prix fabricant hors taxes (PFHT), et non en prix public: ils correspondent donc aux ventes des entreprises pharmaceutiques. Les unités « standard » représentent à la plus petite dose commune d'un produit: comprimé, gélule, ampoule, sachet, etc. Dans la majorité des cas, ces unités standards correspondent donc au conditionnement unitaire que le patient va consommer ou qui va lui être administré.

\section{Données de l'assurance maladie: échantillon généraliste des bénéficiaires}

L'échantillon généraliste des bénéficiaires (ECB) de l'assurance maladie est un échantillon représentatif au I/97e des données du système d'information inter-régime de l'assurance maladie (SnIIRAM)) pour les patients appartenant au régime général et, depuis 20II, pour les patients appartenant à la mutuelle sociale agricole (MSA) et au Régime Social des Indépendants (RSI).

Il intègre tous les bénéficiaires de l'assurance maladie depuis le ler janvier 2003 et permet le suivi, à terme, des patients pendant 20 ans. À la différence du SnIIRAM, il intègre tous les bénéficiaires qu'ils soient consommant ou non. De plus, l'ECB contient des données médicales, au travers des affections de longue durée (ALD30) et données du programme de médicalisation des systèmes d'information (données d'hospitalisation). 
Tous les patients présents dans l'ECB et bénéficiant d'au moins un remboursement d'un traitement de benzodiazépines (anxiolytiques, hypnotiques, anticonvulsivantes et myorelaxantes) à partir du ler juillet 2006 et jusqu'au 30 juin 20llont été sélectionnés, sans autres critères particuliers d'inclusion, ni de non-inclusion.

Les patients ayant changé de régime (MSA et RSI vers le régime général) avant 20Il sont donc intégrés, en plus des nouveaux affiliés aux sections locales mutualistes (SLM, régime des fonctionnaires et des étudiants) depuis 20I0, mais n'ont pas été pris en compte pour l'estimation de la prévalence, limitée aux bénéficiaires affiliés au régime général strict 


\section{Annexe II: Évolution de la consommation des benzodiazépines entre 2000 et 2010 en nombre de DDJ pour 1000 habitants et par jour}

\begin{tabular}{|c|c|c|c|c|c|c|c|c|c|c|c|c|c|}
\hline Code ATC & $\begin{array}{c}\text { Substance } \\
\text { active }\end{array}$ & Com. & 2000 & 2001 & 2002 & 2003 & 2004 & 2005 & 2006 & 2007 & 2008 & 2009 & 2010 \\
\hline $\mathrm{m03B \times 07}$ & Tétrazépam & 1969 & 2,9 & 2,9 & 3,0 & 3,2 & 3,3 & 3,7 & 3,9 & 3,8 & 3,7 & 3,9 & 4,1 \\
\hline ก03AEOI & Clonazépam & 1973 & 1,1 & 1,3 & 1,4 & 1,4 & 1,5 & 1,7 & 1,7 & 1,8 & 1,7 & 1,7 & 1,8 \\
\hline n05CDO2 & nitrazépam & 1966 & 1,1 & 0,9 & 0,8 & 0,7 & 0,6 & 0,6 & 0,5 & 0,4 & 0,4 & 0,4 & 0,3 \\
\hline n05CDO3 & Flunitrazépam & 1978 & 3,8 & 0,9 & 0,6 & 0,5 & 0,4 & 0,4 & 0,4 & 0,3 & 0,2 & 0,2 & 0,2 \\
\hline n05CD04 & Estalozam & 1978 & 0,2 & 0,3 & 0,3 & 0,2 & 0,2 & 0,2 & 0,2 & 0,2 & 0,2 & 0,2 & 0,2 \\
\hline n05CD05 & Triazolam & 1980 & 0,04 & 0,03 & 0,02 & 0,02 & 0,02 & 0,01 & 0,00 & 0,00 & 0,00 & 0,00 & 0,00 \\
\hline n05CD06 & Lormétazépam & 1989 & 4,0 & 4,8 & 5,1 & 5,0 & 5,1 & 5,4 & 5,3 & 5,5 & 5,5 & 5,1 & 5,1 \\
\hline n05CD07 & Témazépam & 1986 & 0,2 & 0,3 & 0,2 & 0,1 & 0,1 & 0,1 & 0,1 & 0,1 & 0,1 & 0,1 & 0,1 \\
\hline n05CD08 & midazolam & 1993 & 0,1 & 0,1 & 0,1 & 0,1 & 0,2 & 0,3 & 0,3 & 0,3 & 0,3 & 0,3 & 0,4 \\
\hline n05CDII & Loprazolam & 1984 & 1,5 & 1,6 & 1,6 & 1,5 & 1,4 & 1,4 & 1,3 & 1,3 & 1,3 & 1,3 & 1,2 \\
\hline nO5CFOI & Zopiclone & 1987 & 10,6 & 10,9 & 11,0 & 9,9 & 11,4 & II,I & 10,9 & 10,4 & 10,0 & 10,0 & 10,3 \\
\hline n05CF02 & Zolpidem & 1988 & 11,6 & 13,4 & 13,9 & 14,5 & 14,5 & 14,4 & 14,4 & 14,8 & 14,5 & 14,8 & 15,3 \\
\hline nО5BAOI & Diazépam & 1966 & 2,4 & 2,4 & 2,3 & 2,3 & 2,3 & 2,6 & 3,0 & 2,6 & 2,6 & 2,7 & 2,8 \\
\hline п05BA04 & Oxazépam & 1966 & 4,8 & 5,1 & 5,4 & 5,1 & 5,4 & 5,0 & 5,4 & 5,7 & 5,9 & 6,2 & 6,5 \\
\hline nО5Вค05 & $\begin{array}{l}\text { Clorazépate } \\
\text { potassique }\end{array}$ & 1968 & 7,2 & 7,3 & 7,3 & 6,8 & 4,6 & 3,9 & 3,0 & 2,7 & 2,5 & 2,4 & 2,3 \\
\hline П05Bम06 & Lorazépam & 1973 & 12,9 & 12,8 & $\mathrm{II}, \mathrm{I}$ & 9,8 & 10,3 & 9,7 & 9,1 & 8,9 & 8,5 & 8,0 & 7,8 \\
\hline П05ВА08 & Bromazépam & 1981 & 11,9 & 12,0 & 12,3 & 11,7 & $\|, 7$ & $\|, 7$ & 11,4 & 11,0 & 10,4 & 10,2 & 10,3 \\
\hline ПО5ВА09 & Clobazam & 1975 & 1,5 & 1,5 & 1,4 & 1,4 & 1,3 & 1,4 & 1,3 & 1,3 & 1,2 & 1,1 & $\mathrm{I}, \mathrm{I}$ \\
\hline n05BAII & Prazépam & 1973 & 3,0 & 3,0 & 3,0 & 2,9 & 3,0 & 3,1 & 3,2 & 3,0 & 3,1 & 2,5 & 3,1 \\
\hline nО5BAI2 & Alprazolam & 1984 & 7,0 & 7,3 & 7,6 & 7,4 & 7,8 & 7,8 & 8,1 & 7,8 & 7,7 & 8,0 & 8,6 \\
\hline nO5BAI6 & nordazépam & 1985 & 0,8 & 0,8 & 0,8 & 0,7 & 0,7 & 0,7 & 0,6 & 0,6 & 0,6 & 0,5 & 0,5 \\
\hline nO5BAI8 & Ethyl loflazépate & 1982 & 1,0 & 1,0 & 1,0 & 0,9 & 0,9 & 0,8 & 0,8 & 0,7 & 0,7 & 0,6 & 0,6 \\
\hline ПО5ВА2І & Clotiazépam & 1986 & 0,3 & 0,3 & 0,3 & 0,3 & 0,3 & 0,3 & 0,3 & 0,3 & 0,3 & 0,3 & 0,2 \\
\hline \multirow[t]{2}{*}{ ПО5ВА23 } & Tofisopam & 1980 & 0,04 & 0,02 & 0,00 & 0,00 & 0,00 & 0,00 & 0,00 & 0,00 & 0,00 & 0,00 & 0,00 \\
\hline & & & 90,0 & 90,8 & 90,5 & 86,5 & 87,1 & 86,1 & 85,2 & \begin{tabular}{|l|}
83,7 \\
\end{tabular} & 81,2 & 81,4 & 82,9 \\
\hline
\end{tabular}

n.B. Le tofisopam n'est plus commercialisé depuis 2001 et le triazolam depuis 2005 


\section{Annexe III : Données issues de l'échantillon généraliste des bénéficiaires}

Table Al. : Répartition démographique des patients sélectionnés

\begin{tabular}{|c|c|c|c|c|}
\hline & $\begin{array}{c}\text { Hypnotiques } \\
n=38976\end{array}$ & $\begin{array}{c}\text { Anxiolytiques } \\
n=87249\end{array}$ & $\begin{array}{c}\text { Clonazépam } \\
\text { n= } 9279\end{array}$ & $\begin{array}{l}\text { Tétrazépam } \\
n=77044\end{array}$ \\
\hline \multicolumn{5}{|l|}{ Âge } \\
\hline$<20$ ans, \% (n) & $2,3(895)$ & $4,5(4321)$ & $3,1(283)$ & 5,1 (3 957) \\
\hline $20-30$ ans, \% (n) & $8,8(3412)$ & II,I (9 699) & $6, I(566)$ & 17,0 (13 07I) \\
\hline $30-40$ ans, $\%$ (n) & $13,2(5$ |28) & 16,4 (14 332) & II,4 (I 053) & 24,0 (I8 49I) \\
\hline $40-50$ ans, \% (n) & 16,7 (6 512) & I8,6 (16 250) & I6,9 (| 57|) & 22,7 (17 499) \\
\hline $50-60$ ans, \% (n) & 19,8 (7 700) & $18,2(15876)$ & 21,3 (| 978) & 17,I (13 193) \\
\hline $60-70$ ans, $\%(n)$ & I5,2 (5 939) & 12,4 (I0 773) & 17,4 (I 614) & $8,3(6405)$ \\
\hline $70-80$ ans, \% (n) & $14,5(5636)$ & II,3 (9 875) & 15,3 (| 417) & 4,5 (3 462) \\
\hline $80-90$ ans, \% (n) & $8,1(3 \mid 56)$ & $6, I$ (5 305) & $7,5(691)$ & ।,2 (912) \\
\hline$\geq 90$ ans, \% (n) & I,5 (598) & $0,9(818)$ & I,I (I06) & $0,1(54)$ \\
\hline Sexe, femmes, \% & 59,1 (23 035) & 65,9 (57 497) & $58,8(5456)$ & $53,0(40833)$ \\
\hline
\end{tabular}

Table All.: Traitement médicamenteux associés et co-morbidités

\begin{tabular}{|c|c|c|c|c|}
\hline & $\begin{array}{l}\text { Hypnotiques } \\
n=38976\end{array}$ & $\begin{array}{l}\text { Anxiolytiques } \\
n=87249\end{array}$ & $\begin{array}{l}\text { Clonazépam } \\
n=9279\end{array}$ & $\begin{array}{l}\text { Tétrazépam } \\
\text { n= } 77044\end{array}$ \\
\hline $\begin{array}{l}\text { Patients ayant au moins } \\
\text { une fLD } 30, \%(n)\end{array}$ & $29,4(25666)$ & 34,5 (13 464) & $38,4(3566)$ & II,4 (8 864) \\
\hline nombre moyen d'ALD30 (SD) & $0,5(0,8)$ & $0,4(0,7)$ & $0,5(0,8)$ & $0,1(0,4)$ \\
\hline \multicolumn{5}{|l|}{ Présence d'une ALD30, \% (n) } \\
\hline Troubles mentaux & $6,6(2584)$ & $7,6(6$ 597) & $5,8(545)$ & I,0 (775) \\
\hline Pathologie tumorale & 9,2 (3 59l) & $7,2(6256)$ & $8,8(820)$ & 2,7 (2 078) \\
\hline Pathologie rhumatologique & I,9 (745) & I,6 (| 410) & 3,I (29) & $0,9(704)$ \\
\hline Diabète type I et II & $6,9(2704)$ & $4,9(4295)$ & 8,6 (793) & $2,6(2008)$ \\
\hline Maladie hypertensive & $5,2(2035)$ & $3,9(3434)$ & $4,9(458)$ & $1,5(1147)$ \\
\hline Pathologie neurologique & $1,9(747)$ & I,9 (I 635) & $4,6(430)$ & $0,6(450)$ \\
\hline $\begin{array}{l}\text { Association à une autre } \\
\text { BZD, \% (n) }\end{array}$ & 31,0 (I2 089) & $28,6(24968)$ & |8,2 (| 69|) & $6,6(5$ II7) \\
\hline $\begin{array}{l}\text { Traitement antipsychotique, } \\
\%(n)\end{array}$ & $8,1(3$ 174) & $8,9(7740)$ & $7,2(670)$ & I,4 (| IOI) \\
\hline $\begin{array}{l}\text { Traitement antidépresseur, } \\
\%(n)\end{array}$ & $25,3(9857)$ & 31,0 (27 042) & $23,5(2$ 179) & $7,5(5766)$ \\
\hline
\end{tabular}

ALD30: affection longue durée appartenant à la liste des 30 maladies exonérées du ticket modérateur. SD : écart-type 
Table All.: Caractéristiques liées à la prescription médicale lors du premier cycle de traitement

\begin{tabular}{|c|c|c|c|c|}
\hline & Hypnotiques & Anxiolytiques & Clonazépam & Tétrazépam \\
\hline Primo-prescription & $\mathrm{n}=38976$ & $n=87249$ & $n=9279$ & $n=77044$ \\
\hline Mode d'exercice libéral, \% (n) & $89,5(34891)$ & $90, I(78693)$ & $84,8(7864)$ & $93,0(71664)$ \\
\hline \multicolumn{5}{|c|}{ Spécialité médicale (si exercice libéral), \% (n) } \\
\hline Médecine générale & $94,0(33134)$ & $91,3(72329)$ & $80,9(6412)$ & $95,1(68418)$ \\
\hline Psychiatrie & $2,3(811)$ & $3,7(2907)$ & $1,7(133)$ & $0,1(34)$ \\
\hline Rhumatologie & $0,3(88)$ & $0,2(176)$ & $5,9(464)$ & $2,0(\mid 462)$ \\
\hline Autres & 3,4 (| 200) & $4,8(3796)$ & $11,6(917)$ & $2,8(2014)$ \\
\hline
\end{tabular}

Table AIV. : Principaux indicateurs liés à la prescription médicale au cours du traitement

\begin{tabular}{|c|c|c|c|c|}
\hline & $\begin{array}{c}\text { Hypnotiques } \\
n=38976\end{array}$ & $\begin{array}{c}\text { Anxiolytiques } \\
n=87249\end{array}$ & $\begin{array}{c}\text { Clonazépam } \\
n=9279\end{array}$ & $\begin{array}{c}\begin{array}{c}\text { Tétrazépam } \\
\mathrm{n}=77044\end{array} \\
\end{array}$ \\
\hline nombre moyen de consultations (SD) & $8,9(\|8\|)$, & $7,2(14,8)$ & $4,4(10,6)$ & $1,5(3,2)$ \\
\hline $\begin{array}{l}\text { nombre moyen de prescripteurs } \\
\text { différents pour se procurer } \\
\text { le traitement (SD) }\end{array}$ & I,5 (I,3) & I,4 (I,I) & I,3 (I,0) & I,I $(0,4)$ \\
\hline nomadisme médical, \% (n) & $4,6(1775)$ & $4,5(3917)$ & $2,7(249)$ & $0,4(275)$ \\
\hline
\end{tabular}

SD: écart-type 
Table AU. : Données relatives à la délivrance pharmaceutique de traitement par benzodiazépines et apparentées

\begin{tabular}{|c|c|c|c|c|}
\hline & $\begin{array}{l}\text { Hypnotiques } \\
n=38976\end{array}$ & $\begin{array}{c}\text { Anxiolytiques } \\
n=87249\end{array}$ & $\begin{array}{l}\text { Clonazépam } \\
n=9279\end{array}$ & $\begin{array}{l}\text { Tétrazépam } \\
\text { n= } 77044\end{array}$ \\
\hline $\begin{array}{l}\text { Patients ne bénéficiant que } \\
\text { d'une seule déliurance, } \% \text { (n) }\end{array}$ & $51,0(19874)$ & $51,7(45064)$ & $55,2(5 \mid 25)$ & $81,6(62836)$ \\
\hline $\begin{array}{l}\text { nombre médian } \\
\text { de déliurances (si 2), (IQR) }\end{array}$ & $9(3-28)$ & $9(3-32)$ & $5(3-14)$ & $2(2-4)$ \\
\hline $\begin{array}{l}\text { Délai médian entre } \\
2 \text { déliurances (IQR), mois }\end{array}$ & ।,3 (I,0-1,9) & I,I (I,0-I,5) & I,3 (I,0-1,8) & I,I (0,6-I,8) \\
\hline \multicolumn{5}{|c|}{ Patients bénēficiant d'une déliurance selon l'âge, \% (n) } \\
\hline$<30$ ans $(n=28967)$ & $15,9(3 \mid 55)$ & $22,9(10341)$ & $11,5(587)$ & $23,7(14884)$ \\
\hline $30-50$ ans $(n=55704)$ & $35,3(7023)$ & $39,5(17776)$ & $30,9(\mid 583)$ & $46,7(29322)$ \\
\hline $50-70$ ans $(n=35504)$ & $33,3(6622)$ & $26,0(11717)$ & $36,8(1887)$ & $24,3(15278)$ \\
\hline$>70$ ans $(n=12724)$ & $15,5(3074)$ & $11,6(5230)$ & 20,8 (। 068) & $5,3(3352)$ \\
\hline $\begin{array}{l}\text { Temps d'exposition médian } \\
\text { (IQR), mois }\end{array}$ & $7,2(2,4-30,1)$ & $6,7(2,2-30,5)$ & $4,0(1,8-13,5)$ & I,8 (0,8-3,2) \\
\hline $\begin{array}{l}\text { Traitement de plus de } 3 \\
\text { mois, \% (n) }\end{array}$ & $73.9(28800)$ & $70.5(6 \mid 525)$ & $67.0(6215)$ & 49.8 (38 333) \\
\hline \multicolumn{5}{|c|}{ Traitement de plus de 3 mois selon l'âge, \% (n) } \\
\hline$<30$ ans $(n=36204)$ & $52.3(2254)$ & $48,2(6.755)$ & $50.5(429)$ & $39.4(6712)$ \\
\hline $30-50$ ans $(n=80836)$ & $71.5(8317)$ & $69.6(21280)$ & 67.9 (। 782) & 53.5 (19 255) \\
\hline $50-70$ ans $(n=63478)$ & 78.7 (I0 729) & 77.7 (20694) & $70.6(2537)$ & $52.6(10316)$ \\
\hline$>70$ ans $(n=32030)$ & $79.9(7500)$ & $80.0(12796)$ & $66.3(\mid 467)$ & $46.3(2050)$ \\
\hline $\begin{array}{l}\text { Posologie > Dose max Amm*, } \\
\%(n)\end{array}$ & $19,4(3706)$ & $16,0(6742)$ & I2,2 (506) & 23,7 (3 360) \\
\hline
\end{tabular}

SD: écart-type, IQR: intervalle interquartile

* Amm: autorisation de mise sur le marché, proportion estimée pour les patients bénéficiant de plus 2 délivrances et à partir de la dose maximale utilisée en psychiatrie pour chaque benzodiazépine

Table AVI. : Proportion de sujets exposés aux benzodiazépines en fonction du temps de traitement (total ou continu) et de l'indication

\begin{tabular}{|l|c|c|c|c|c|c|c|c|}
\hline \multirow{2}{*}{ Tps d'exposition } & \multicolumn{2}{|c|}{ Anxiolytiques } & \multicolumn{2}{c|}{ Hypnotiques } & \multicolumn{2}{c|}{ Clonazépam } & \multicolumn{2}{c|}{ Tétrazépam } \\
\cline { 2 - 10 } & $n$ & $\%$ & $n$ & $\%$ & $n$ & $\%$ & $n$ & $\%$ \\
\hline moins de 3 mois & 25724 & 29.5 & 10176 & 26.1 & 3064 & 33.0 & 38711 & 50.2 \\
\hline 3 - 6 mois & 3024 & 3.5 & 1401 & 3.6 & 363 & 3.9 & 2446 & 3.2 \\
\hline 6 - 9 mois & 2609 & 3.0 & 1129 & 2.9 & 307 & 3.3 & 2304 & 3.0 \\
\hline 9 - I2 mois & 2258 & 2.6 & 1123 & 2.9 & 254 & 2.7 & 2120 & 2.8 \\
\hline $12-24$ mois & 9478 & 10.9 & 4490 & 11.5 & 1026 & 11.1 & 8363 & 10.9 \\
\hline $24-36$ mois & 8704 & 10.0 & 4052 & 10.4 & 914 & 9.9 & 7866 & 10.2 \\
\hline 36 - 48 mois & 9830 & 11.3 & 4718 & 12.1 & 992 & 10.7 & 8115 & 10.5 \\
\hline $48-60$ mois & 25622 & 29.4 & 11887 & 30.5 & 2359 & 25.4 & 7119 & 9.2 \\
\hline
\end{tabular}


Table AVII. : Caractéristiques d'un traitement par benzodiazépine pendant le suivi des patients

\begin{tabular}{|c|c|c|c|c|}
\hline & $\begin{array}{l}\text { Hypnotiques } \\
n=38976\end{array}$ & $\begin{array}{c}\text { Anxiolytiques } \\
n=87249\end{array}$ & $\begin{array}{l}\text { Clonazépam } \\
\text { n=9279 }\end{array}$ & $\begin{array}{l}\text { Tétrazépam } \\
n=77044\end{array}$ \\
\hline $\begin{array}{l}\text { Temps de suivi médian (IQR), } \\
\text { mois }\end{array}$ & $43,7(16,3-58,2)$ & $44,8(16,0-58,8)$ & $38,4(11,0-57,9)$ & $19,0(2,4-42,4)$ \\
\hline $\begin{array}{l}\text { nb moyen de BZD différentes } \\
\text { tout au long de la période } \\
\text { (SD) }\end{array}$ & $2,5(1,5)$ & $2,3(1,5)$ & $2,3(1,4)$ & $2,0(1,2)$ \\
\hline $\begin{array}{l}\text { Patient débutant au moins } \\
\text { un nouveau traitement, \% (n) }\end{array}$ & 57,2 (22 284) & $54,7(47688)$ & $57,3(5316)$ & 47,9 (36 927) \\
\hline $\begin{array}{l}\text { Temps médian de survenue } \\
\text { d'un nouveau traitement } \\
\text { [IC95], mois }\end{array}$ & $14,7[14,3-15,0]$ & $16,9[|6,6-17|]$, & $16,9[15,9-17,6]$ & $29,8[29,4-30,2]$ \\
\hline $\begin{array}{l}\text { Probabilité de reprendre } \\
\text { un traitement à } 24 \text { mois }\end{array}$ & 0,598 & 0,569 & 0,572 & 0,433 \\
\hline
\end{tabular}

SD: écart-type; IC95: Intervalle de confiance à $95 \%$ 
Annexe IV: niveaux de risque pour la conduite automobile, attribués aux benzodiazépines

lextrait de l'arrêté du 8 août 2008, pris pour l'application de l'article R. 5121-139 du Code de la santé publique, qui donne la liste complète de toutes les substances actives possédant des effets sur les capacités à conduire des véhicules ou à utiliser des machines).

\begin{tabular}{|c|c|}
\hline Alprazolam & niveau 2 \\
\hline Bromazépam & niveau 2 \\
\hline Brotizolam & niveau 3 \\
\hline Clobazam & niveau 2 \\
\hline Clonazépam & $\begin{array}{l}\text { niveau } 2 \text { (formes orales) } \\
\text { niveau } 3 \text { (formes parentérales) }\end{array}$ \\
\hline Clorazépate & $\begin{array}{c}\text { niveau } 2 \text { (formes orales) } \\
\text { niveau } 3 \text { (formes parentérales et formes orales dosées à } 20 \mathrm{mg} \text { et plus) }\end{array}$ \\
\hline Chlordiazépoxide & niveau 2 \\
\hline Clotiazépam & niveau 2 \\
\hline Diazépam & $\begin{array}{c}\text { niveau } 2 \text { (formes orales) } \\
\text { niveau } 3 \text { (formes parentérales et formes orales dosées à l0 mg) }\end{array}$ \\
\hline Estazolam & niveau 3 \\
\hline Flunitrazépam & niveau 3 \\
\hline Flurazépam & niveau 3 \\
\hline Loflazépate & niveau 2 \\
\hline Loprazolam & niveau 3 \\
\hline Lorazépam & $\begin{array}{c}\text { Miveau } 2 \\
\text { niveau } 3 \text { (formes à } 2,5 \mathrm{mg} \text { ) }\end{array}$ \\
\hline Lormétazépam & niveau 3 \\
\hline médazépam & niveau 2 \\
\hline Midazolam & niveau 3 \\
\hline nitrazépam & niveau 3 \\
\hline nordazépam & $\begin{array}{c}\text { niveau } 2 \\
\text { niveau } 3 \text { (formes à } 15 \mathrm{mg} \text { ) }\end{array}$ \\
\hline Oxazépam & $\begin{array}{c}\text { Miveau } 2 \\
\text { niveau } 3 \text { (formes à } 50 \mathrm{mg} \text { ) }\end{array}$ \\
\hline Prazépam & $\begin{array}{c}\text { niveau } 2 \\
\text { niveau } 3 \text { (formes à } 40 \mathrm{mg} \text { ) }\end{array}$ \\
\hline Témazépam & niveau 3 \\
\hline Tétrazépam & niveau 2 \\
\hline Tofisopam & niveau 2 \\
\hline Triazolam & niveau 3 \\
\hline Zolpidem & niveau 3 \\
\hline Zopiclone & niveau 3 \\
\hline
\end{tabular}

Pictogrammes associés au niveau de risque pour la conduite automobile:
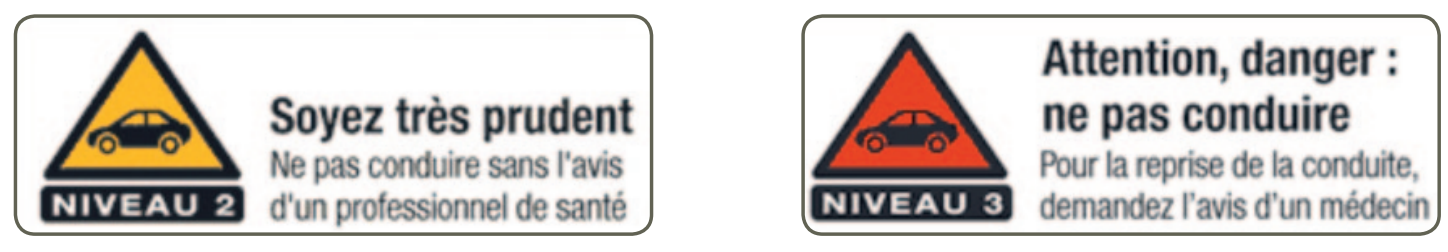


\section{Annexe U}

\section{- Flunitrazépam (Rohypnolø)}

\begin{tabular}{|c|c|}
\hline $\begin{array}{l}\text { Risques identifiés } \\
\text { par le réseau } \\
\text { d'addictovigilance }\end{array}$ & $\begin{array}{l}\text { Années } 1990 \\
\text { - abus et de détournement important chez les toxicomanes } \\
\text { - soumission chimique +++ }\end{array}$ \\
\hline $\begin{array}{l}\text { Actions mises en œuvre } \\
\text { par l'Afssaps }\end{array}$ & $\begin{array}{l}\text { - } 1996 \text { : retrait du Rohypno|}\left.\right|^{\circledR} 2 \mathrm{mg} \\
\text { - } 1996 \text { : restriction de l'utilisation de l'indication thérapeutique du Rohypnol } \\
\text { I mg aux « troubles sévères du sommeil » } \\
\text { - } 1999 \text { : modification de la galénique } \\
\text { - } 1999 \text { : réduction de la taille du conditionnement } \\
\text { - } 2001 \text { : prescription sur ordonnance sécurisée et durée maximale de } \\
\text { prescription limitée à } 14 \text { jours avec une délivrance fractionnée de } 7 \text { jours }\end{array}$ \\
\hline $\begin{array}{l}\text { Impact des différentes } \\
\text { mesures }\end{array}$ & $\begin{array}{l}\text { Sur la consommation: } \\
\text { - Entre } 1997 \text { et } 1999 \text { : diminution de } 40 \text { \% de la consommation du Rohypno }{ }^{\oplus} \\
\text { I mg } \\
\text { - Entre } 2000 \text { et } 2009 \text { : chute des ventes de près de } 94 \% \\
\text { - Estimation du nombre de patients en } 2010 \text { : } 13349 \mid \\
\text { Sur l'abus et l'usage détourné chez les toxicomanes: } \\
\text { - Diminution significative du nombre de cas d’abus et de détournement } \\
\text { - Persistance de l'abus chez des anciens consommateurs: ler rang en termes } \\
\text { d'indicateurs d'abus } \\
\text { Sur la soumission chimique: } \\
\text { - Plus d'utilisation du flunitrazépam depuis la modification galénique } \\
\text { du comprimé } \\
\text { Sur le bon usage: } \\
\text { - Respect non systématique des conditions de prescription et de délivrance }\end{array}$ \\
\hline Actions à mener & $\begin{array}{l}\text { - Réaliser des actions de communication sur le bon usage du flunitrazépam, } \\
\text { notamment auprès de l'Assurance maladie et des Conseils de l'Ordre des } \\
\text { médecins et des pharmaciens } \\
\text { - Maintien de la surveillance par le réseau des CEIP }\end{array}$ \\
\hline
\end{tabular}

\section{- Clorazépate dipotassique (Tranxène ${ }^{\oplus}$ )}

\begin{tabular}{|l|l|}
\hline $\begin{array}{l}\text { Risques identifiés } \\
\text { par le réseau } \\
\text { d'addictovigilance }\end{array}$ & $\begin{array}{l}\text { Enquête d'addictovigilance en } 2003 \\
\text { - abus et de détournement important chez les toxicomanes +++ }\end{array}$ \\
\hline $\begin{array}{l}\text { Actions mises en œuuvre } \\
\text { par l'Afssaps }\end{array}$ & $\begin{array}{l}\text { - } 2003 \text { : prescription du Tranxène }{ }^{\oplus} 50 \mathrm{mg} \text { sur ordonnance sécurisée et durée } \\
\text { maximale de prescription et de délivrance limitée à } 28 \text { jours } \\
\text { - } 2005 \text { : retrait du Tranxène } 50 \mathrm{mg} \text { et mise sur le marché du Tranxène }{ }^{\oplus} 20 \mathrm{mg} \\
\text { - } 2005 \text { : prescription du Tranxène } 20 \mathrm{mg} \text { sur ordonnance sécurisée et durée } \\
\text { maximale de prescription et de déliurance limitée à } 28 \text { jours }\end{array}$ \\
\hline $\begin{array}{l}\text { Impact des différentes } \\
\text { mesures }\end{array}$ & $\begin{array}{l}\text { Les mesures mises en œuure ont eu un impact positif: les indicateurs de } \\
\text { détournement et d'abus ont nettement diminué. }\end{array}$ \\
\hline Actions à mener & - Maintien de la surveillance par le réseau des CEIP \\
\hline
\end{tabular}


- Zopiclone (Stilnox $\left.{ }^{\circledast}\right)$

\begin{tabular}{|c|c|}
\hline $\begin{array}{l}\text { Risques identifiés par } \\
\text { le réseau d'addictovigilance }\end{array}$ & $\begin{array}{l}\text { Enquête } 2002 \text { : } \\
\text { - Mise en évidence d'un risque d’abus et de dépendance au sein de } 2 \text { types } \\
\text { de population: } \\
\text { - dépendance et abus chez des personnes consommant le zolpidem } \\
\quad \text { dans une finalité hypnotique } \\
\text { - dépendance et abus chez des personnes consommant le zolpidem } \\
\text { pour rechercher des effets psychiques positifs } \\
\text { Enquête } 2011 \text { couvrant la période 2003/I0: } \\
\text { - Confirmation de ces deux types de population } \\
\text { - Mise en évidence d'un profil particulier du zolpidem par rapport aux autres } \\
\text { bzd: apparition d'effets paradoxaux non observés avec les autres bzd } \\
\text { et cas de dépendance de sévérité importante (consommation de doses } \\
\text { particulièrement élevées) }\end{array}$ \\
\hline $\begin{array}{l}\text { Actions mises en œuure } \\
\text { par l'Afssaps }\end{array}$ & $\begin{array}{l}\text { - } 2004 \text { : modification du RCP: ajout du risque « Pharmacodépendance » } \\
\text { et renforcement de la rubrique « Mise en garde et précautions d'emploi » }\end{array}$ \\
\hline $\begin{array}{l}\text { Impact des différentes } \\
\text { mesures }\end{array}$ & $\begin{array}{l}\text { La modification du RCP en } 2004 \text { n'a pas eu d'impact. } \\
\text { L'actualisation de l'enquête d'addictovigilance montre: } \\
\text { - la persistance des risques identifiés en } 2002 \text {, avec une aggravation des cas } \\
\text { de dépendance: } 2^{e} \text { rang pour l'indicateur « Dose/2 Amm »; } \\
\text { - la caractérisation d'un profil particulier du zolpidem (effets paradoxaux+++); } \\
\text { - le non-respect des conditions de prescription et de délivrance }\end{array}$ \\
\hline Actions à mener & $\begin{array}{l}\text { - Mieux encadrer la prescription du zolpidem: proposition de rendre } \\
\text { obligatoire la prescription du zolpidem sur ordonnance sécurisée } \\
\text { - Informer l'Assurance maladie sur l'émergence de l'abus de zolpidem } \\
\text { afin de renforcer sa surveillance } \\
\text { - Demander au laboratoire des données sur la pharmacologie et la } \\
\text { pharmacodynamie du zolpidem } \\
\text { - Maintien de la surveillance par le réseau des CEIP }\end{array}$ \\
\hline
\end{tabular}

À souligner que les différents outils du réseau d'addictovigilance ne mettent pas en évidence en 20।0 de signaux particuliers pour le zopiclone (lmovane ${ }^{\oplus}$ l, contrairement au zolpidem.

\section{- Anxiolytiques \\ Alprazolam}

\begin{tabular}{|l|l|}
\hline $\begin{array}{l}\text { Risques identifiés par } \\
\text { le réseau d'addictovigilance }\end{array}$ & $\begin{array}{l}\text { - Risque d'abus et de détournement en augmentation (toxicomanes et } \\
\text { patients âgés présentant des comorbidités psychiatriques): I ler rang pour } \\
\text { l'indicateur « Souffrance à l'arrêt » } \\
\text { - Recours comme produit de coupage à l'héroïne +++ }\end{array}$ \\
\hline Actions à mener & Maintien de la surveillance par le réseau des CEIP \\
\hline
\end{tabular}


Bromazépam

\begin{tabular}{|c|c|}
\hline $\begin{array}{l}\text { Risques identifiés par } \\
\text { le réseau d'addictovigilance }\end{array}$ & $\begin{array}{l}\text { - Risques d'abus et de détournement important: } 3^{e} \text { rang pour la majorité } \\
\text { des indicateurs de détournement } \\
\text { - Soumission chimique : } 2^{\mathrm{e}} \text { rang parmi les benzodiazépines +++ }\end{array}$ \\
\hline $\begin{array}{l}\text { Actions mises en œuvre } \\
\text { par l'Afssaps }\end{array}$ & $\begin{array}{l}\text { - } 2004 \text { : modification du RCP: ajout du risque « Pharmacodépendance » et } \\
\text { renforcement de la rubrique « Mise en garde et précautions d'emploi » }\end{array}$ \\
\hline Actions à mener & - Maintien de la surveillance par le réseau des CEIP \\
\hline
\end{tabular}

\section{Clonazépam (Rivotril $\left.{ }^{\circledR}\right)$}

Le clonazépam est indiqué dans la prise en charge de l'épilepsie. Toutefois, les différentes enquêtes d’addictovigilance ont montré que le clonazépam est prescrit dans près de $90 \%$ dans d'autres indications, en particulier dans la prise en charge des douleurs mais également dans diverses pathologies.

\begin{tabular}{|c|c|}
\hline $\begin{array}{l}\text { Risques identifiés par } \\
\text { le réseau d'addictovigilance }\end{array}$ & $\begin{array}{l}\text { - Abus et détournement en particulier chez les toxicomanes } \\
\text { - Soumission chimique: }\left.\right|^{\text {er }} \text { rang/les benzodiazépines +++ } \\
\text { - Prescription hors-Amm ( } 90 \% \text { des cas) ++++ } \\
\text { - Émergence d'un trafic à destination de pays tiers (Afrique du nord) }\end{array}$ \\
\hline $\begin{array}{l}\text { Actions mises en œuvre } \\
\text { par l'Afssaps }\end{array}$ & $\begin{array}{l}\text { - } 2008 \text { : mise en place d’un PGR national } \\
\text { - réduction du conditionnement } \\
\text { - lettre aux prescripteurs sur le bon usage } \\
\text { - ajout d'un colorant dans la solution buvable pour limiter le risque } \\
\text { de soumission chimique } \\
\text { - 20I0: restriction de la durée maximale de prescription à I2 semaines } \\
\text { - 20II : prescription sur ordonnance sécurisée }\end{array}$ \\
\hline $\begin{array}{l}\text { Impact des différentes } \\
\text { mesures }\end{array}$ & $\begin{array}{l}\text { Les mesures mises en place entre } 2008 \text { et } 2010 \text { n'ont pas eu d'impact } \\
\text { sur les différents risques identifiés. Le clonazépam est en } 2^{e} \text { position pour } \\
3 \text { indicateurs de détournement. Le clonazépam demeure prescrit dans } \\
\text { près de } 90 \% \text { des cas en dehors des indications de l'Amm. } \\
\text { En outre, depuis } 2010 \text {, il a été constaté une augmentation du trafic } \\
\text { du Rivotril }{ }^{\otimes} \text {,en particulier de la forme comprimé, reposant essentiellement } \\
\text { sur la falsification d'ordonnances. }\end{array}$ \\
\hline Actions à mener & $\begin{array}{l}\text { - Maintien de la surveillance par le réseau des CEIP et l'Assurance maladie } \\
\text { - Mise au point sur l'arrêt de l'utilisation hors Amm du clonazépam } \\
\text { notamment dans la douleur, les troubles anxieux et les troubles } \\
\text { du sommeil (fin 20II) } \\
\text { - Restriction de la prescription initiale réservée aux neurologues et pédiatres } \\
\text { à partir de janvier } 2012\end{array}$ \\
\hline
\end{tabular}





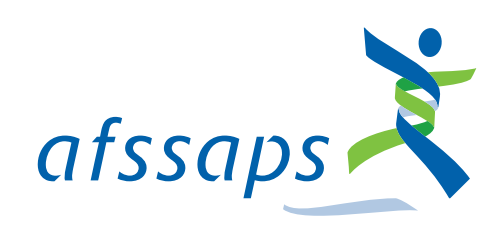

Agence française de sécurité sanitaire des produits de santé

L'Agence française de sécuritē sanitaire des produits de santé 143/I47, boulevard Anatole France - F-93285 Saint-Denis Cedex - têl. +33 (0) I 55873000 - fax +33 (0) I 558730 I2 www.afssaps.fr 